\title{
About a Partial Differential Equation-Based Interpolator for Signal Envelope Computing: Existence Results and Applications
}

\author{
Oumar Niang, ${ }^{1,2,3}$ Abdoulaye Thioune,, Éric Deléchelle, ${ }^{2}$ \\ Mary Teuw Niane, ${ }^{3}$ and Jacques Lemoine ${ }^{2}$ \\ ${ }^{1}$ Département Informatique et Télécommunications, Ecole Polytechnique de Thiès (EPT), Thiès BP A10, Senegal \\ ${ }^{2}$ Laboratoire Images, Signaux et Systèmes Intelligents (LISSI-E.A.3956), Université Paris-Est Créteil Val-de-Marne, Créteil, France \\ ${ }^{3}$ Laboratoire d'Analyse Numérique et d'Informatique (LANI), Université Gaston Berger (UGB), Saint-Louis BP 234, Senegal \\ ${ }^{4}$ Département de Mathématique et Informatique, Faculté des Sciences et Technique, Université Cheikh Anta Diop de Dakar, \\ Dakar BP 5005, Senegal
}

Correspondence should be addressed to Oumar Niang; oniang@ucad.sn

Received 6 November 2012; Accepted 3 December 2012

Academic Editors: H. Hu and S. Li

Copyright (C) 2013 Oumar Niang et al. This is an open access article distributed under the Creative Commons Attribution License, which permits unrestricted use, distribution, and reproduction in any medium, provided the original work is properly cited.

This paper models and solves the mathematical problem of interpolating characteristic points of signals by a partial differential Equation-(PDE-) based approach. The existence and uniqueness results are established in an appropriate space whose regularity is similar to cubic spline one. We show how this space is suitable for the empirical mode decomposition (EMD) sifting process. Numerical schemes and computing applications are also presented for signal envelopes calculation. The test results show the usefulness of the new PDE interpolator in some pathological cases like input class functions that are not so regular as in the cubic splines case. Some image filtering tests strengthen the demonstration of PDE interpolator performance.

\section{Introduction}

Interpolators are widely used in signal and processing or data analysis. In particular for the empirical mode decomposition (EMD) algorithm $[1,2]$, the iterative estimation of the signal trend is based on the computing of the envelopes obtained by the cubic spline interpolation of local extrema. The spline interpolation has been recognized as being very effective for EMD. But for signals that have no local extremum, the cubic spline interpolation fails. We proposed a PDE-based model which overcomes this limit of classical EMD implementation, in the computing of the envelopes for signals that have no local extremum [2-4]. Recently, the Spectral Intrinsic Decomposition (SID) method [5], based on the spectral decomposition of the PDE interpolator, provides a new application of our model. This PDE interpolator contribute, to the mathematical modeling of the EMD and has provided various applications in signal and image processing $[4,6]$. In this paper, we describe the mathematical modeling of the new PDE interpolator by variational methods. The resolution of the variational problem leads to existence and uniqueness results in appropriate spaces. The paper is organized as follows. In Sections 2 and 2.1 recalls some PDE models in signal and image processing, and in Section 2.2 some mathematical preliminary notions are set out. In Section 3, the mathematical modeling is exposed. In Sections 4 and 5, the resolution of the variational problem is dealt. Subsequently, numerical implementation and applications are presented in Section 6. At last, we finish by conclusions.

\section{Some Preliminaries}

\subsection{PDE Models in Signal and Image Processing and EMD Principle}

2.1.1. Some Diffusion Equations. This part consists of a brief and nonexhaustive presentation of classical nonlinear diffusion filters for $1 \mathrm{D}$ and $2 \mathrm{D}$ signal processing, with more focus on the $2 \mathrm{D}$ case. In this purpose, we can recall the model for nonlinear diffusion in image filtering proposed by Catte et al. [7]. This filter is a modified version of the well-know 
Perona and Malik model [8]. The basic equation that governs nonlinear diffusion filtering is

$$
u_{t}(\mathbf{x}, t)=\operatorname{div}\left(g\left(|\nabla u(\mathbf{x}, t)|^{2}\right) \nabla u(\mathbf{x}, t)\right)
$$

with $\mathbf{x}=\left(x_{1}, x_{2}\right)$, and where $u(\mathbf{x}, t)$ is a filtered version of the original image $u(\mathbf{x}, t)=u_{0}(\mathbf{x})$ as the initial condition, with reflecting boundary conditions. In $(1), g(\cdot)$ is the conductivity (or diffusivity) function, which is dependent (in space and time) on the image gradient magnitude. Several forms of diffusivity were introduced in the original paper of Perona and Malik [8]. All forms of diffusivity are chosen to be a monotonically decreasing function of the signal gradient. Possible expressions for conductivity are

$$
\begin{gathered}
g_{1}(\mathbf{x}, t)=\frac{1}{1+(|\nabla u(\mathbf{x}, t)| / \beta)^{2}}, \\
g_{2}(\mathbf{x}, t)=\exp \left(-(|\nabla u(\mathbf{x}, t)| / \beta)^{2}\right) .
\end{gathered}
$$

Parameter $\beta$ is a threshold one, which influences the anisotropic smoothing process. The nonlinear equation (1) acts as a forward parabolic equation smoothing regions while preserving edges. Other methods based on high-order PDE are provided for image restoration like in [9-11]. Efficient numerical schemes were introduced in [12] based on additive operator splitting (AOS) scheme, or based on alternating direction implicit (ADI) scheme. See [12-15] for a review and extensions of these methods. Unlike these methods of highorder PDE that are specially developed for denoising, our model was constructed to interpolate the characteristic points of a signal.

The major problem of nonlinear diffusion-based process is that it is generally difficult to correctly separate the high frequency components from the low frequency ones. In case of denoising applications, the objective of this process is to use the diffusivity function as a guide to retain useful data and suppress noise.

Numerous authors have proposed fourth-order PDEs for image smoothing and denoising with the hope that these methods would perform better than their second-order analogues [16-18]. Indeed, there are good reasons to consider fourth-order equations. The first reason that can be retained is the fact that fourth-order linear diffusion damps oscillations at high frequencies (e.g., noise) much faster than secondorder diffusion. On the other hand, the theory of fourth order nonlinear PDEs is far less developed than the second order one. Also such equations often do not satisfy a maximum principle or comparison principle, and implementation of the equations could thus introduce artificial singularities or other undesirable behavior. In recent studies, Tumblin [19], Tumblin and Turk [16], and Wei [17] proposed the following form:

$$
u_{t}(\mathbf{x}, t)=-\operatorname{div}(g(m(u)) \nabla \Delta u(\mathbf{x}, t))
$$

where $g(\cdot)=g_{1}(\cdot)$ as in (2) and $m$ is some measurement of $u(\mathbf{x}, t)$. In [16], (3) is called a "low curvature image simplifier" (LCIS), and a good choice for $m$ is defined as $m=\Delta u$ to enforce isotropic diffusion [19]. These PDE tools for digital signal and image processing make more reachable the $2 \mathrm{D}$ extension of the 1D PDE-based method for characteristic points interpolation presented in this paper.

2.1.2. The Empirical Mode Decomposition Principle. The EMD [1] method decomposes iteratively a signal into amplitude modulation-frequency modulation (AM-FM) type components called intrinsic mode functions (IMF). The underlying principle of this decomposition is to locally identify in the signal, the most rapid oscillations defined as the waveform interpolating interwoven local maxima and minima. To do this, local extrema points are interpolated with a cubic spline, to yield the upper and lower envelopes. The mean envelope (half sum of upper and lower envelopes) is then subtracted from the initial signal, and the same interpolation scheme is reiterated on the remainder. The so-called sifting process stops when the mean envelope is reasonably zero everywhere, and the resulting signal is called the first IMF. The higher-order IMFs are iteratively extracted applying the same procedure to the initial signal after the previous IMFs have been removed.

2.2. Some Useful Mathematical Concepts. Throughout the paper, $\Omega$ denotes an open-bounded subset of $\mathbb{R}^{n}, n=1$ or 2 . In the sequel, we will need the following definitions and results.

Definition 1. we define one the spaces $V$ and $\mathscr{V}$ as follows

$$
\begin{gathered}
V=\left\{v \in H^{3}(\Omega)\left|\frac{\partial v}{\partial v}\right|_{\partial \Omega}=0\right\}, \\
\mathscr{V}=\left\{v \in H^{3}(\Omega)\left|\frac{\partial v}{\partial v}\right|_{\partial \Omega}=0, \int_{\Omega} v d x=0\right\}, \\
\mathscr{V}_{e}=\left\{v \in H^{3}(\Omega) \text { such that } \exists \Gamma_{\Omega} \subset \Omega,\right. \\
\text { open and verifying } \left.\int_{\Gamma_{\Omega}} v d x=0 \text { and }\left.\frac{\partial v}{\partial v}\right|_{\partial \Omega}=0\right\} .
\end{gathered}
$$

Definition 2. For a given function $v \in H^{1}(\Omega)$, if $D_{d}(v)(x)$ and $D_{g}(v)(x)$ denote, respectively, the right and left derivative of $v$ at $x$; the minmod derivatives of $v$ is define by

$$
\begin{array}{r}
\frac{\partial v}{\partial x}(x)=\operatorname{minmod}\left(D_{g}(v)(x), D_{d}(v)(x)\right) \\
\operatorname{minmod}(p, q)= \begin{cases}\operatorname{sign}(p) \min (|p|,|q|), & \text { if } p q>0, \\
0, & \text { if } p q \leq 0 .\end{cases}
\end{array}
$$

A $H^{1}(\Omega)$ function being absolutely continuous admits right and left derivatives, then $u_{0} \in H^{1}(\Omega)$ has obviously left and right derivatives, so that we can validate numerically computing of the diffusivity function $g$ defined in Section 3. 
Definition 3. Let $\left(x_{n}\right)_{n \in \mathbb{N}}$ be a sequence of elements in a vectorial normed space $\left(E,\|\cdot\|_{E}\right)$; it is said that $\left(x_{n}\right)_{n \in \mathbb{N}}$ converge, weakly [20] in $E$, and noted by $x_{n} \rightarrow x$, if exists an element $x \in E$ such that $\forall f \in E^{\prime}, \lim _{n \rightarrow \infty} f\left(x_{n}\right)=f(x)$, where $E^{\prime}$ denotes the set of continuous linear forms on $E$.

Definition 4 (the Green formula). Let $u, v \in C^{2}(\Omega)$, then

$$
\begin{gathered}
\int_{\Omega} \Delta u d x=\int_{\partial \Omega} \frac{\partial u}{\partial n} d s \\
\int_{\Omega} \nabla u \nabla v d x=-\int_{\Omega} u \Delta v d x+\int_{\partial \Omega} \frac{\partial v}{\partial n} d s \\
\int_{\Omega}(u \Delta v-v \Delta u) d x=\int_{\partial \Omega} u \frac{\partial v}{\partial n}-v \frac{\partial u}{\partial n} d s \text { Green formula, }
\end{gathered}
$$

where $\partial u / \partial n$ denotes the normal derivative of $u$ on the boundary $\partial \Omega$ of $\Omega$.

Definition 5 (the Poincaré-Wirtinger inequality). Let $\Omega$ be an open-bounded set, and let $u \in H^{1}(\Omega)$, then there exists a constant $C>0$ such that the norm of $u$ in $H^{1}(\Omega)$ and the norm of $u$ in $L^{2}(\Omega)$ are linked by the following inequality:

$$
\left\|u-\frac{1}{|\Omega|} \int_{\Omega} u d s\right\|_{H^{1}(\Omega)} \leq C\|\nabla u\|_{L^{2}(\Omega)},
$$

where $|\Omega|$ denotes the length of $\Omega$. The best constant $C$ in the Poincaré-Wirtinger inequality is $1 / \lambda$, for example, the inverse of the first positive eigenvalue $\lambda$ of the Laplace operator with homogenous Neumann boundaries conditions. In our case, $\Omega \subset \mathbb{R}$, and $|\Omega|$ is the diameter of $\Omega$.

Theorem 6 (regularity theorem). Let

$$
u \in\left\{u \in H^{3}(\Omega) \text { such that }\left.\frac{\partial u}{\partial n}\right|_{\partial \Omega}=0, \int_{\Omega} u d x=0\right\} \text {, }
$$

then there exist two strictly positive reals $C$ and $K$ such that firstly,

$$
\|\nabla u\|_{L^{2}(\Omega)}^{2} \leq C\|\Delta u\|_{L^{2}(\Omega)}^{2},
$$

and secondly,

$$
\|u\|_{H^{3}(\Omega)}^{2} \leq K\|\Delta u\|_{H^{1}(\Omega)}^{2} .
$$

The main existence and uniqueness result of the solution of our henceforth problem (29) is due to the application of the following Lions theorem [21].

Theorem 7 (the Lions theorem). Let $H$ and $V$ be two Hilbert spaces with $V \subset H$. One considers a bilinear application a on $V \times V$ and $B$ an operator on $V$. Under the following conditions:

(1) $\exists \mu$ and $\rho>0$ such that $|a(u, v)| \geq \rho\|v\|_{V}^{2}-\mu|v|_{H}^{2}$,

(2) $\exists c_{2}>0$ such that $\left|(v, B v)_{V}\right| \leq c_{2}|v|_{V}^{2}$.

$$
\begin{aligned}
& \text { For } u_{0}=u(0) \in H \text {, the problem } \\
& \qquad u \in L^{2}(0, T ; V) \cap C(0, T ; H),
\end{aligned}
$$

such that

$$
\forall v \in V, \quad\left(\frac{d u}{d t}, B v\right)_{H}+a(u, v)=0
$$

and $u_{0}=u(0)$, has a unique solution.

Furthermore, $d u / d t \in L^{2}\left(0, T ; H^{\prime}\right)$.

\section{Mathematical Modeling of the New PDE-Interpolator}

Recall that the PDE model aimed to contribute to the mathematical modeling of the EMD, as it is in [2]. In a first step, to be in line with the classical EMD, our goal is to model the upper or lower envelope as the asymptotic solution of a PDE system whose initial condition is the input signal that we want to interpolate local extrema (or more generally characteristic points). Initially, this envelope is obtained by cubic spline interpolation. Let $\Omega$ be the open domain of an given finite energy signal $u_{0} \in L^{2}(\Omega)$. We construct an operator $A$ of appropriate domain $D(A)$ as follows:

$$
\begin{array}{r}
\frac{\partial u}{\partial t}+A u=0 \quad \text { in }[0, T] \times \Omega, \\
u(0, x)=u_{0} \quad \text { in } \Omega,
\end{array}
$$

plus boundaries conditions.

The need of asymptotic solution existence denoted by $u_{\infty}$ implies $(\partial u / \partial t)(\infty, x)=0$, for example, according to (13), we have $A u(\infty, x)=0$. Moreover, the requirement of the same regularity between the solution and a cubic spline leads to $\partial^{4} u / \partial x^{4}(\infty, x)=0$. Thus, in the first analysis, we can choose $A=\partial^{4} u / \partial x^{4}$. To leave invariant features points during the diffusion process, simply multiply in the expression $A u$ the term $\partial^{4} u / \partial x^{4}$ by function $g$ depending on spatial variable $x$ and vanishing at characteristic points. That gives

$$
(A u)(x)=g(x) \frac{\partial^{4} u}{\partial x^{4}}
$$

Another reminiscent form of long-range diffusion [22] is the following:

$$
(A u)(x)=\frac{\partial}{\partial x}\left(g(x) \frac{\partial^{3} u}{\partial x^{3}}\right)
$$

Function $g$ can be interpreted as a diffusivity function whose role is to control the diffusion process. We take it necessarily positive to have a direct diffusion and the existence of solution. Other forms of operators are possible [3] we present some ones as follows: for (14)

$$
(A u)(x)=g(x)\left[-\theta \frac{\partial^{2} u}{\partial x^{2}}+(1-\theta) \frac{\partial^{4} u}{\partial x^{4}}\right],
$$


or for (15),

$$
(A u)(x)=\frac{\partial}{\partial x}\left[g(x)\left(-\theta \frac{\partial u}{\partial x}+(1-\theta) \frac{\partial^{3} u}{\partial x^{3}}\right)\right],
$$

or accomplete diffusion involving a flow

$$
(A u)(x)=\frac{\partial}{\partial x}\left[-\theta g(x) \frac{\partial u}{\partial x}+(1-\theta) \frac{\partial}{\partial x}\left(g(x) \frac{\partial^{2} u}{\partial x^{2}}\right)\right],
$$

where $0 \leq \theta \leq 1$ is the tension factor which controls the regularity of the solution.

Both forms allow more freedom on the regularity of the solution $u$. To access the mathematical properties of the solution, we chose the second form in (17) with $\theta=0$ and the corresponding equation (15).

At the local scale, between two consecutive characteristic points-two local maxima, for example-the diffusion induces a smoothing phenomenon that deletes the local minimum. A simple form for $g$ to calculate the envelope is given by a positive piecewise function lower than 1 that is constant between two characteristic points of $u_{0}$ and zeroed only at these points. Characteristic points are often being defined by their values and the signs of first, second, and third local derivatives of $u_{0}$. We characterize the function $g$ as depending on $\operatorname{sign}\left(\partial u_{0} / \partial x\right), \operatorname{sign}\left(\partial^{2} u_{0} / \partial x^{2}\right)$, and $\operatorname{sign}\left(\partial^{3} u_{0} / \partial x^{3}\right)$.

For the purpose of existence and regularity of the solution, we are led to work with the regularized version of sign:

$$
\operatorname{sign}_{\vartheta}(z)=\frac{2}{\pi} \arctan \left(\frac{\pi z}{\vartheta}\right),
$$

where $\vartheta$ is a regularization coefficient.

In the following, we define $g_{e}^{ \pm}$for extrema detection, $g_{f}$ for turning points detection, and $g_{\mathrm{mc}}^{ \pm}$for maximum and minimum curvature points detection:

$$
g_{e}^{ \pm}(x)=\frac{1}{9}\left[\left|\operatorname{sign}\left(\frac{\partial u_{0}}{\partial x}\right)\right| \pm \operatorname{sign}\left(\frac{\partial^{2} u_{0}}{\partial x^{2}}\right)+1\right]^{2}
$$

(+) for maxima and (-) for minima,

$$
\begin{gathered}
g_{f}(x)=\left[\operatorname{sign}_{\vartheta}\left(\frac{\partial^{2} u_{0}}{\partial x^{2}}\right)\right]^{2} \text { for turning points, } \\
g_{\mathrm{mc}}^{ \pm}(x)=\frac{1}{9}\left[\left|\operatorname{sign}\left(\frac{\partial^{3} u_{0}}{\partial x^{3}}\right)\right| \pm \operatorname{sign}\left(\frac{\partial^{2} u_{0}}{\partial x^{2}}\right)+1\right]^{2},
\end{gathered}
$$

$(+)$ for maximum curvature points and $(-)$ for minimum curvature points. All these functions are of the form $g(x)=$ $[h(x)]^{2}$ with $h(x)=0$ at characteristic points.

So, we have $g^{\prime}(x)=0$ if $g(x)=0$. This property formally allows us to cancel $A u$ and $\partial u / \partial t$ of the form (15) at the points where $g$ is null. The factor $1 / 9$ is a normalization term and verifies $0 \leq g(x) \leq 1$. A more general diffusivity function $g$ could be envisaged.

Let us consider the following differential operators $A_{e}$ and $A_{f}$ ( $e$ for upper and $f$ for inflexion or curvature):

$$
\forall u \in D\left(A_{e}\right), \quad A_{e} u=\frac{\partial}{\partial x}\left(g_{e}^{ \pm}(x) \frac{\partial^{3} u}{\partial x^{3}}\right),
$$

or

$$
\forall u \in D\left(A_{f}\right), \quad A_{f} u=\frac{\partial}{\partial x}\left(g_{f}(x) \frac{\partial^{3} u}{\partial x^{3}}\right) .
$$

If $u_{0} \in C^{2}(\Omega)$, as it is the case in the classical implementation EMD, there is no problem for interpolation by cubic spline method. But it is indeed a restriction on the regularity of $u_{0}$ which is due to the choice of the interpolation technique that requires a good detection of local extrema for the envelopes calculation. The basic EMD principle must apply for input functions that are not regular as functions in $C^{2}(\Omega)$, for example, for signal $u_{0} \in H^{1}(\Omega)$, as it is the case in reality [23]. But numerically, without even a regularization function sign, if the derivatives are taken in the sense of minmod called flux limiter or slope, the function $g(x)$ still has a meaning.

\subsection{Interpretation of the Diffusivity Action in the Diffusion} Process. Between two consecutive maxima $X_{\max _{i}}$ and $X_{\max _{i+1}}$, the function $g_{e}^{+}$is piecewise constant and can be written as follows:

$$
g(x)= \begin{cases}0, & \text { at } X_{\max _{i}}, X_{\max _{i+1}}, \\ \frac{4}{9}, & \text { at } X_{\mathrm{inf}_{i}}, X_{\mathrm{inf}_{i+1}}, X_{\min _{i}}, \\ 1, & \text { on }] X_{\mathrm{inf}_{i}}, X_{\min _{i}}[\cup] X_{\min _{i}}, X_{\inf _{i+1}}[ \\ \frac{1}{9}, & \text { sur }] X_{\max _{i}}, X_{\inf _{i}}[\cup] X_{\inf _{i+1}}, X_{\max _{i+1}}[.\end{cases}
$$

We have a diffusion in the sense that $u_{t}=\partial u / \partial t$ unhook to the maximum of the curve $u_{0}$. The reason is that the diffusion is more pronounced in local minimum because the smoothing effect tends to regularize curves. Thus, the upper envelope $u(t \cong \infty, \cdot)$ of $u_{0}$ is less oscillating than $u_{0}$ is. The same behavior occurs for the mean envelope. We would then try to interpret locally the relationship $u \geq u_{0}$ by evoking the maximum principle [24]. But, this principle is not immediately checked for equations of the type (15). The justification lies in the fact that smoothing implies $\|\nabla u(t, \cdot)\|_{L^{2}(\Omega)} \leq\left\|\nabla u_{0}\right\|_{L^{2}(\Omega)}$.

In summary, we have just built a PDE system

(1) having the input signal $u_{0}$ to decompose as an initial value condition,

(2) such that the result of a diffusion process preserves characteristic points of $u_{0}$,

(3) with a regularity similar to a cubic spline.

3.2. Formulation of the Mathematical Problem. Let $A$ be one of the above constructed interpolation operators, and $T>0$ be a diffusion time sufficiently long. To fix ideas, we solve the problem of upper envelope calculus. Indeed, for the other kinds of characteristic points, the same problem arises with analogous mathematical resolution methods. 
The problem posed by our mathematical modeling is to find a solution $u$ of the system

$$
\begin{array}{r}
\frac{\partial u}{\partial t}+A u=0 \quad \text { in }[0, T] \times \Omega, \\
u(0, x)=u_{0} \quad \text { in } \Omega,
\end{array}
$$

plus boundaries conditions.

Then, the mean envelope (interpolating turning points) calculus is stated as follows.

For given $u_{0}$, find $u$ such that

$$
\begin{array}{r}
\frac{\partial u}{\partial t}+\frac{\partial}{\partial x}\left(g_{f}(x) \frac{\partial^{3} u}{\partial x^{3}}\right)=0 \quad \text { in }[0, T] \times \Omega, \\
u(0, x)=u_{0} \quad \text { in } \Omega,
\end{array}
$$

plus boundaries conditions.

3.3. Comments on the Degeneracy in Problem (24). To prevent the zero degeneracy case in the system (25) whose solution is not directly accessible by variational methods, we are led to solve an intermediate problem with

$$
0<\alpha \leq g_{f, \alpha}(x) \leq \beta \leq 1
$$

That is the nondegenerate problem defined hereafter.

3.4. Formulation of the Zero Degeneracy Induced by Diffusivity Function. The diffusivity function in (26) is very close to zero at the characteristic points while refraining to cancel and to solve the system (25), now, just to move on to the limit when $\alpha$ tends to zero to retrieve (25) and its solution.

Thus, in particular, we can define the function

$$
g_{f, n}(x)=g_{f}(x)+\frac{1}{n}, \quad n \in \mathbb{N}^{*}
$$

which verifies the conditions of (26).

The new system that we call nondegenerate problem is fomulated as follows.

Find $u_{n}$ such that

$$
\begin{array}{r}
\frac{\partial u_{n}}{\partial t}+\frac{\partial}{\partial x}\left(g_{n}(x) \frac{\partial^{3} u_{n}}{\partial x^{3}}\right)=0 \quad \text { in }[0, T] \times \Omega, \\
u_{n}(0, x)=u_{0} \quad \text { in } \Omega,
\end{array}
$$

plus boundaries conditions.

3.5. Formulation of the Nondegenerate Problem. For $n$ sufficiently large, $u_{n}$ is a close approximation of the envelope $u$, interpolating the characteristic points.

By a density technique, we can demonstrate that this sequence $\left(u_{n}\right)_{n \in \mathbb{N}}$ converges to the solution of the system (25). By posing for the convenience of notation $0<\alpha \leq g_{f, n}=h \leq$ $\beta \leq 1$, the non-degenerate problem is reworded as.
Find $u$ such that

$$
\begin{array}{r}
\frac{\partial u}{\partial t}+\frac{\partial}{\partial x}\left(h(x) \frac{\partial^{3} u}{\partial x^{3}}\right)=0 \quad \text { in }[0, T] \times \Omega, \\
u(0, x)=u_{0} \quad \text { in } \Omega,
\end{array}
$$

plus boundaries conditions.

Equation (29) is an operational differential equations type. The techniques for the resolution of this kind of problem are numerous we mainly refer to the variational formulation to find weak solutions [25] and the method of the approximation of evolutionary operators. The last allows to work on a new elliptic problem. The solution is obtained by passing to the limit of the approximate solution nstead of the equation.

In the next section, we solve (29) by a variational approach, according to the resolution methods for parabolic problems described in $[7,20]$.

\section{Existence and Uniqueness of Solutions for PDE Interpolator}

4.1. The Guideline for the Resolution of the Mathematical Problem. Now, we summarize the general procedure to solve the problem.

(1) First step: resolution of the problem (29). This step includes

(i) the variational formulation in Section 4.3,

(ii) the resolution of the variational problem in appropriate functional space,

(iii) the converse showing that the solution of the variational problem solves the departure problem in Section 4.3.4.

(2) Second step: in Section 5, construction of the sequence $\left(u_{n}\right)_{n \in \mathbb{N}}$ of solutions of problem (29), where $u_{n}$ is the solution obtained with the diffusivity. $h_{n}(x)=$ $h(x)+(1 / n), n \in \mathbb{N}^{*}$, see Section 5. Finally, demonstrate that the sequence $\left(u_{n}\right)_{n \in \mathbb{N}}$ converges to a limit $u$, where $u$ is the solution of problem (25), in Section 5.

4.2. The Main Results Formulation. The main result which is going to be solves is the following.

Theorem 8. For $u_{0} \in H^{1}(\Omega)$, exists a unique solution $u \in$ $L^{2}(0, T ; \mathscr{V}) \cap C\left([0, T] ; H^{1}(\Omega)\right)$ of the variational problem

$$
\frac{d}{d t} \int_{\Omega} \frac{\partial u}{\partial x} \frac{\partial v}{\partial x} d x+\int_{\Omega} h(x) \frac{\partial^{3} u}{\partial x^{3}} \frac{\partial^{3} v}{\partial x^{3}} d x=0, \quad \text { for any } v \in \mathscr{V}
$$

such that $u(0)=u_{0}$ and $d u / d t \in L^{2}(0, T ; H)$. Reversely, let $u$ be a solution of the variational problem (30), then $(\partial u / \partial t)+$ $(\partial / \partial x)\left(h(x)\left(\partial^{3} u / \partial x^{3}\right)\right)=0$.

And the complete follows result. 
Theorem 9. For $u_{0} \in H^{1}(\Omega)$, exists a unique solution

$$
u \in L^{2}(0, T ; \mathscr{V}) \cap C\left([0, T] ; H^{1}(\Omega)\right),
$$

verifying

$$
\begin{gathered}
\frac{\partial u}{\partial t}+\frac{\partial}{\partial x}\left(h(x) \frac{\partial^{3} u}{\partial x^{3}}\right)=0 \quad \text { in }[0, T] \times \Omega, \\
\left.\frac{\partial^{3} u}{\partial x^{3}}\right|_{\partial \Omega}=0 \quad(\text { on boundaries of } \Omega), \\
\left.\frac{\partial u}{\partial n}\right|_{\partial \Omega}=0 \quad(\text { on boundaries of } \Omega), \\
u(0, x)=u_{0} \quad \text { in } \Omega .
\end{gathered}
$$

4.3. Variational Formulation. Let $v \in H^{3}(\Omega)$, and (to make sense to integrals) multiply the equation

$$
\frac{\partial u}{\partial t}+\frac{\partial}{\partial x}\left(h(x) \frac{\partial^{3} u}{\partial x^{3}}\right)=0
$$

of the system (29) by the test function $\partial^{2} v / \partial x^{2}$ after we integrate on $\Omega$, then it comes out that

$$
\int_{\Omega} \frac{d u}{d t} \frac{\partial^{2} v}{\partial x^{2}} d x+\int_{\Omega} \frac{\partial}{\partial x}\left(h(x) \frac{\partial^{3} u}{\partial x^{3}}\right) \frac{\partial^{2} v}{\partial x^{2}} d x=0 .
$$

By using the Green formula (see Definition 4) and integration by parts, we get firstly

$$
\begin{aligned}
\int_{\Omega} & \frac{\partial}{\partial x}\left(h(x) \frac{\partial^{3} u}{\partial x^{3}}\right) \frac{\partial^{2} v}{\partial x^{2}} d x \\
\quad & {\left[h(x) \frac{\partial^{3} u}{\partial x^{3}} \frac{\partial^{2} v}{\partial x^{2}}\right]_{\partial \Omega}-\int_{\Omega} h(x) \frac{\partial^{3} u}{\partial x^{3}} \frac{\partial^{3} v}{\partial x^{3}} d x }
\end{aligned}
$$

and secondly,

$$
\begin{gathered}
\int_{\Omega} \frac{d u}{d t} \frac{\partial^{2} v}{\partial x^{2}} d x=\frac{d}{d t} \int_{\Omega} u \frac{\partial^{2} v}{\partial x^{2}} d x \\
\int_{\Omega} u \frac{\partial^{2} v}{\partial x^{2}} d x=\int_{\partial \Omega} u \frac{\partial v}{\partial n} d \sigma-\int_{\Omega} \frac{\partial u}{\partial x} \frac{\partial v}{\partial x} d x
\end{gathered}
$$

As we are in one-dimension case, the normal derivative and the gradient on the edge in the use of the Green formula are the same, that is to say,

$$
\int_{\partial \Omega} u \frac{\partial v}{\partial n} d \sigma=\int_{\partial \Omega} u \frac{\partial v}{\partial x} d \sigma
$$

In the following, we adopt the notation $\partial v / \partial n$.

Considering $u \in H^{3}(\Omega)$ such that

$$
\begin{gathered}
\left.\frac{\partial^{3} u}{\partial x^{3}}\right|_{\partial \Omega}=0 \\
v \in V=\left\{v \in H^{3}(\Omega)\left|\frac{\partial v}{\partial n}\right|_{\partial \Omega}=0\right\}
\end{gathered}
$$

it comes out that

$$
\frac{d}{d t} \int_{\Omega} \frac{\partial u}{\partial x} \frac{\partial v}{\partial x} d x+\int_{\Omega} h(x) \frac{\partial^{3} u}{\partial x^{3}} \frac{\partial^{3} v}{\partial x^{3}} d x=0,
$$

$\forall v \in V$.

Let us consider the following bilinear forms

$$
\begin{gathered}
a(u, v)=\int_{\Omega} h(x) \frac{\partial^{3} u}{\partial x^{3}} \frac{\partial^{3} v}{\partial x^{3}} d x \\
b(u, v)=\int_{\Omega} \frac{\partial u}{\partial x} \frac{\partial v}{\partial x} d x
\end{gathered}
$$

The addition of boundaries conditions transforms our initial problem into the system

$$
\begin{gathered}
\frac{\partial u}{\partial t}+\frac{\partial}{\partial x}\left(h(x) \frac{\partial^{3} u}{\partial x^{3}}\right)=0 \quad \text { in }[0, T] \times \Omega, \\
\left.\frac{\partial^{3} u}{\partial x^{3}}\right|_{\partial \Omega}=0 \quad(\text { on the edge of } \Omega), \\
\left.\frac{\partial u}{\partial n}\right|_{\partial \Omega}=0 \quad(\text { on the edge of } \Omega), \\
u(0, x)=u_{0} \quad \text { in } \Omega .
\end{gathered}
$$

The variational formulation of our problem consequently, subject to additional conditions that would cause a change of space, is the following

$$
\text { Find } u \in V \text { such that } \frac{d}{d t}(b(u, v))+a(u, v)=0 \text {, }
$$

$\forall v \in V$,

where $a$ and $b$ are like in Theorem 7 .

This kind of problem is studied in [21]. However, before continuing the resolution, we explore some properties of the space $V$.

4.3.1. On the Quotient Space $V / \mathbb{R}$. The space $V$ is defined by $D(a) \cap D(b)$, where $D(a)$ and $D(b)$ are the domains of $a$ and $b$. Let us consider the quotient space

$$
\begin{gathered}
\frac{V}{\mathbb{R}}=\left\{\{v+c, c \in \mathbb{R}\} \text { such that } v \in H^{3}(\Omega),\right. \\
\text { and } \left.\left.\frac{\partial v}{\partial n}\right|_{\partial \Omega}=0\right\}
\end{gathered}
$$

and the new space $\mathscr{V}$ defined by

$$
\mathscr{V}=\left\{v \in H^{3}(\Omega)\left|\frac{\partial v}{\partial n}\right|_{\partial \Omega}=0, \int_{\Omega} v d x=0\right\} .
$$

Let $\widetilde{v} \in V / \mathbb{R}$, and let $u$ be a representative of $\widetilde{v}$ class, then $\left.(\partial \widetilde{v} / \partial n)\right|_{\partial \Omega}$ is defined by

$$
\left.\frac{\partial \widetilde{v}}{\partial n}\right|_{\partial \Omega}=\left.\frac{\partial(u+c)}{\partial n}\right|_{\partial \Omega}=\left.\frac{\partial u}{\partial n}\right|_{\partial \Omega}=0 \quad \text { with } c \in \mathbb{R} .
$$


The nullity of $\int_{\Omega} \widetilde{v} d x$ means $\exists c \in \mathbb{R}$, constant such that $\int_{\Omega}(u+$ c) $d x=0$, with $u$ one representative of $\widetilde{v}$

$$
\begin{gathered}
\text { if } c=-\frac{1}{|\Omega|} \int_{\Omega} u d x, \text { then } \\
\int_{\Omega} \widetilde{v} d x=\int_{\Omega}(u+c) d x \\
=\int_{\Omega}\left(u-\frac{1}{|\Omega|} \int_{\Omega} u d x\right) d x=0 .
\end{gathered}
$$

So for all $\tilde{v} \in V / \mathbb{R}$, we have $\int_{\Omega} \tilde{v} d x=0$. Accordingly, any element of the quotient space $V / \mathbb{R}$ is, on one hand, in $H^{3}(\Omega)$ with normal derivative on the boundaries of $\Omega$ null and, on the other hand, is zero average on $\Omega$. Thus, the quotient space $V / \mathbb{R}$ is identified with the space $\mathscr{V}$. We can work later in the space $\mathscr{V}$ with zero mean. The final variational problem to solve is the following

$$
\text { find } u \in \mathscr{V} \text { such that } \frac{d}{d t}(b(u, v))+a(u, v)=0, \quad \forall v \in \mathscr{V} \text {. }
$$

4.3.2. Application of the Lions Theorem. To apply the Lions theorem, we need to identify the applications $a, b$, and $B$ and the spaces $H$ and $V$. And finally, we must prove that the norm defined in $\mathscr{V}$, which derives from the scalar product given by the bilinear application $a$, is equivalent to the norm of $H^{3}(\Omega)$.

Let us define $B$ as the identity, and let us define $a$ and $b$ by $|a(u, v)|=\left(\sqrt{h}\left(\partial^{3} u / \partial x^{3}\right), \sqrt{h}\left(\partial^{3} v / \partial x^{3}\right)\right)_{L^{2}(\Omega)}$, and $b(u, v)=$ $\int_{\Omega}(\partial u / \partial x)(\partial v / \partial x) d x$, obtained from (39), (40); the spaces $V=\mathscr{V}$ and $H$ are define as follows.

(a) The linear operator $B$ : in the variational problem (11) in the Lions theorem, $(d u / d t, B v)_{H}$ denotes a scalar product on $H$. Indeed, we define $H=\{v \in$ $H^{1}(\Omega)$ such that $\left.\left.(\partial v / \partial n)\right|_{\partial \Omega}=0, \int_{\Omega} v d x=0\right\}$.

The operator $B=I, B v=v$, is the identity which is continuous (verify the Lions conditions Theorem 7), and the term $(d u / d t, B v)_{H}=(d / d t)(b(u, v))$ is equal to

$$
\begin{aligned}
\int_{\Omega} \frac{\partial}{\partial x}\left(\frac{d u}{d t}\right) \frac{\partial v}{\partial x} d x & =\int_{\Omega} \frac{d}{d t}\left(\frac{\partial u}{\partial x}\right) \frac{\partial v}{\partial x} d x \\
& =\left(\frac{\partial}{\partial x}\left(\frac{d u}{d t}\right), \frac{\partial v}{\partial x}\right)_{L^{2}(\Omega)}
\end{aligned}
$$

In this identification, $((\partial / \partial x)(d u / d t), \partial v / \partial x)_{L^{2}(\Omega)}$ is a well scalar product in the subspace $H$ of $H^{1}(\Omega)$, formed by functions with zero mean. Indeed, according to PoincareWirtinger inequality (see Definition 5) in $H^{1}(\Omega)$,

$$
\left\|u-\frac{1}{|\Omega|} \int_{\Omega} u d x\right\|_{H^{1}(\Omega)} \leq \frac{1}{\lambda}\left\|\frac{\partial u}{\partial x}\right\|_{L^{2}(\Omega)},
$$

where $\lambda$ is the first positive eigenvalue of Laplace operator in $\Omega$. But as $u, v \in \mathscr{V}$, then $\int_{\Omega} u d x=0$, consequently there exists $C_{2}>0$ such that:

$$
\|u\|_{H^{1}(\Omega)} \leq C_{2}\left\|\frac{\partial u}{\partial x}\right\|_{L^{2}(\Omega)},
$$

that is to say the $H^{1}(\Omega)$ norm is equivalent to the gradient norm in $\mathscr{V}$. Hence, one can replace the scalar product of the gradient by $H^{1}(\Omega)$ scalar product.

Hereinafter, we complete the proof that the precedent choices allow the application of the Lions theorem in our context.

(b) $\mathscr{V}$ is an Hilbert space with equivalent norm to $H^{3}(\Omega)$ norm.

Theorem 10. The space $\mathscr{V}$, resulting from (11) and $\mathscr{V}$ analysis, defined by

$$
\mathscr{V}=\left\{v \in H^{3}(\Omega)\left|\frac{\partial v}{\partial n}\right|_{\partial \Omega}=0, \int_{\Omega} v d x=0\right\}
$$

is an Hilbert space with the a norm equivalent to $H^{3}(\Omega)$ norm.

Proof. At first $\mathscr{V}=\left\{v \in H^{3}(\Omega)|(\partial v / \partial n)|_{\partial \Omega}=0, \int_{\Omega} v d x=\right.$ $0\}$ is a closed vector subspace of $H^{3}(\Omega)$. Note that the condition of the nullity of the mean in $\mathscr{V}$ may be replaced by $\int_{\Gamma_{\Omega}} u d x=0$, where $\Gamma_{\Omega}$ is a nonempty open subset of $\Omega$. In this case $\mathscr{V}$ becomes rather

$$
\begin{gathered}
\mathscr{V}_{e}=\left\{v \in H^{3}(\Omega) \text { such that } \exists \Gamma_{\Omega} \subset \Omega\right. \text { verifying } \\
\left.\int_{\Gamma_{\Omega}} v d x=0 \text {, and }\left.\frac{\partial v}{\partial n}\right|_{\partial \Omega}=0\right\} .
\end{gathered}
$$

From the fact that

$$
0<\alpha \leq h(x) \leq \beta<1, \quad \forall x \in \Omega,
$$

the norms built with the two bilinear applications $a$ and $b$ are equivalent to the norm constructed by the linear form, noted by $|\|\||_{\mathscr{V}}^{2}$ with $v \in \mathscr{V}$, and given by

$$
|\|v\||_{\mathscr{V}}^{2}=\left\|\frac{\partial v}{\partial x}\right\|_{L^{2}(\Omega)}^{2}+\left\|\frac{\partial^{3} v}{\partial x^{3}}\right\|_{L^{2}(\Omega)}^{2} .
$$

Indeed, $|\|\||_{\mathscr{V}}^{2}$ is as well a norm in $\mathscr{V}$. There is no difficulty to formally verify the triangular inequality. If $|\|v\||_{\mathscr{V}}^{2}=0$, then $\|\partial v / \partial x\|_{L^{2}(\Omega)}^{2}=0$ and $\left\|\partial^{3} v / \partial x^{3}\right\|_{L^{2}(\Omega)}^{2}=0$. Consequently, $v$ is necessarily constant almost every where in $\Omega$, for example, $v$ is equal to a constant real $K$. But as $\int_{\Omega} v d x=0$ implies $K|\Omega|=$ 0 , so $K=v=0$. We have thus built a standard scalar product on $\mathscr{V}$ from bilinear forms of the variational formulation. $\mathscr{V}$ is a Hilbert space with the scalar product associated with the norm (54). In addition, this norm is equivalent to the $H^{3}(\Omega)$ norm, as it shown in Lemma A.2. Now, we just need to prove that the correspondence $(u, v) \in \mathscr{V} \times \mathscr{V} \mapsto a(u, v)$ is continuous and coercive on the Hilbert space $\mathscr{V}$. 
4.3.3. The Application $a(\cdot, \cdot)$ Is Continuous and Coercive. For $u, v \in \mathscr{V}$,

$$
\begin{aligned}
|a(u, v)| & =\left|\left(\sqrt{h} \frac{\partial^{3} u}{\partial x^{3}}, \sqrt{h} \frac{\partial^{3} v}{\partial x^{3}}\right)_{L^{2}(\Omega)}\right| \\
& \leq \beta\left\|\frac{\partial^{3} u}{\partial x^{3}}\right\|_{L^{2}(\Omega)} \cdot\left\|\frac{\partial^{3} v}{\partial x^{3}}\right\|_{L^{2}(\Omega)} \\
& \leq \beta\left|\| u \| \left\|_{\mathscr{V}} \cdot|\|v\||_{\mathscr{V}},\right.\right.
\end{aligned}
$$

hence the continuity of $a(\cdot, \cdot)$ is on $\mathscr{V} \times \mathscr{V}$. In order to complete the application conditions of Theorem 7 , we only check the first condition of the same theorem.

For

$$
v \in \mathscr{V}, \quad-\|v\|_{H^{1}(\Omega)}^{2} \leq-\left\|\frac{\partial v}{\partial x}\right\|_{L^{2}(\Omega)}^{2},
$$

hence

$$
|\|v\||_{\mathscr{V}}^{2}-\|v\|_{H^{1}(\Omega)}^{2} \leq|\|v\||_{\mathscr{V}}^{2}-\left\|\frac{\partial v}{\partial x}\right\|_{L^{2}(\Omega)}^{2},
$$

nevertheless

$$
\begin{aligned}
a(v, v) & =\left\|\sqrt{h} \frac{\partial^{3} v}{\partial x^{3}}\right\|_{L^{2}(\Omega)}^{2} \geq \alpha\left\|\frac{\partial^{3} v}{\partial x^{3}}\right\|_{L^{2}(\Omega)}^{2} \\
& =\alpha\left[\mid\|v\|\left\|_{\mathscr{V}}^{2}-\right\| \frac{\partial v}{\partial x} \|_{L^{2}(\Omega)}^{2}\right] .
\end{aligned}
$$

Thus,

$$
a(v, v) \geq \alpha\left[|\|v\||_{\mathscr{V}}^{2}-\|v\|_{H^{1}(\Omega)}^{2}\right], \quad \forall v \in \mathscr{V} .
$$

We have found thus $(\rho, \mu)=(\alpha, \alpha)$ such that

$$
a(v, v) \geq \rho|\|v\||_{\mathscr{V}}^{2}-\mu\|v\|_{H^{1}(\Omega)}^{2}, \quad \forall v \in \mathscr{V} .
$$

All the conditions of Theorem 7 are acquired; now, the main existence result for solution of system (41) can be enunciated as follows.

Theorem 11. For $u_{0} \in H^{1}(\Omega)$, exists a unique solution $u \in$ $L^{2}(0, T ; \mathscr{V}) \cap C\left([0, T] ; H^{1}(\Omega)\right)$ of the problem

$$
\begin{array}{r}
\frac{d}{d t} \int_{\Omega} \frac{\partial u}{\partial x} \frac{\partial v}{\partial x} d x+\int_{\Omega} h(x) \frac{\partial^{3} u}{\partial x^{3}} \frac{\partial^{3} v}{\partial x^{3}} d x=0, \\
\text { for any } v \in \mathscr{V} \text { such that } \\
u(0)=u_{0}, \quad \frac{d u}{d t} \in L^{2}(0, T ; H) .
\end{array}
$$

4.3.4. Equivalence with the Initial Problem. Reversely, let $u$ be a solution of the variational problem

$$
\frac{d}{d t} \int_{\Omega} \frac{\partial u}{\partial x} \frac{\partial v}{\partial x} d x+\int_{\Omega} h(x) \frac{\partial^{3} u}{\partial x^{3}} \frac{\partial^{3} v}{\partial x^{3}} d x=0 \quad \forall v \in \mathscr{V}
$$

As we must consider a test function

$$
v \in H^{3}(\Omega) \text { such that } \frac{\partial^{2} v}{\partial x^{2}} \text { still in } D(\Omega),\left.\quad \frac{\partial v}{\partial v}\right|_{\partial \Omega}=0
$$

Let us take a function test $\phi \in D(\Omega)$ and $K \in \mathbb{R}$ such that

$$
\int_{\Omega}(\phi+K) d x=0
$$

We pose the following Neumann problem:

$$
\begin{gathered}
\frac{\partial^{2} v}{\partial x^{2}}=\phi+K \\
\left.\frac{\partial v}{\partial n}\right|_{\partial \Omega}=0 .
\end{gathered}
$$

Its variational formulation gives a unique solution in $H=$ $\left\{w \in H^{1}(\Omega) \mid \int_{\Omega} w d x=0\right\}$, and the regularity of $\phi \in H^{2}(\Omega)$ implies the regularity of the solution which is in $v \in H^{3}(\Omega)$, consequently $v \in \mathscr{V}$.

Returning to (62) with $v$, solution of the problem (65), the first term can be written as

$$
\begin{aligned}
& \frac{d}{d t} \int_{\Omega} \frac{\partial u}{\partial x} \frac{\partial v}{\partial x} d x=\frac{d}{d t}\left(\int_{\Omega} u \frac{\partial^{2} v}{\partial x^{2}} d x-\int_{\partial \Omega} u \frac{\partial v}{\partial n} d \sigma\right), \\
& \text { as } \frac{\partial^{2} v}{\partial x^{2}}=\phi+K,\left.\quad \frac{\partial v}{\partial n}\right|_{\partial \Omega}=0
\end{aligned}
$$

and it comes out that

$$
\begin{aligned}
\frac{d}{d t} \int_{\Omega} \frac{\partial u}{\partial x} \frac{\partial v}{\partial x} d x & =\frac{d}{d t} \int_{\Omega} u(\phi+K) d x \\
& =\frac{d}{d t}\left(\int_{\Omega} u \phi d x+K \int_{\Omega} u d x\right) .
\end{aligned}
$$

However, $\int_{\Omega} u d x=0$, subsequently

$$
\frac{d}{d t} \int_{\Omega} \frac{\partial u}{\partial x} \frac{\partial v}{\partial x} d x=\frac{d}{d t} \int_{\Omega} u \phi d x=\int_{\Omega} \frac{d u}{d t} \phi d x
$$

The second term of (62) is written as follows:

$$
\int_{\Omega} h(x) \frac{\partial^{3} u}{\partial x^{3}} \frac{\partial^{3} v}{\partial x^{3}} d x=\int_{\Omega} h(x) \frac{\partial^{3} u}{\partial x^{3}} \frac{\partial \phi}{\partial x} d x
$$

which gives in the distribution sense

$$
\int_{\Omega} h(x) \frac{\partial^{3} u}{\partial x^{3}} \frac{\partial^{3} v}{\partial x^{3}} d x=\int_{\Omega} \frac{\partial}{\partial x}\left(h(x) \frac{\partial^{3} u}{\partial x^{3}}\right) \phi d x
$$

from which

$$
\int_{\Omega} \frac{d u}{d t} \phi d x+\int_{\Omega} \frac{\partial}{\partial x}\left(h(x) \frac{\partial^{3} u}{\partial x^{3}}\right) \phi d x=0, \quad \forall \phi \in D(\Omega) .
$$


We deduce that

$$
\frac{d u}{d t}+\frac{\partial}{\partial x}\left(h(x) \frac{\partial^{3} u}{\partial x^{3}}\right)=0
$$

We verify now that $\left.\left(\partial^{3} u / \partial x^{3}\right)\right|_{\partial \Omega}=0$. Returning to (70) with $\phi=\partial^{2} v / \partial x^{2}$ and according to the Green formula, we have

$$
\begin{aligned}
\int_{\Omega}\left[\frac{d u}{d t}+\frac{\partial}{\partial x}\left(h(x) \frac{\partial^{3} u}{\partial x^{3}}\right)\right] \frac{\partial^{2} v}{\partial x^{2}} d x \\
-\int_{\partial \Omega} h(x) \frac{\partial^{3} u}{\partial x^{3}} \frac{\partial}{\partial n}\left(\frac{\partial v}{\partial x}\right) \cdot n d \sigma=0
\end{aligned}
$$

$\forall v \in \mathscr{V}$

But as $(\partial / \partial n)(\partial v / \partial x) \cdot n=(\partial / \partial x)(\partial v / \partial x)=\partial^{2} v / \partial x^{2}$, it gives according to (71) $\int_{\partial \Omega} h(x)\left(\partial^{3} u / \partial x^{3}\right)\left(\partial^{2} v / \partial x^{2}\right) d \sigma=0 \forall v \in$ $\mathscr{V}$, and consequently $h(x)\left(\partial^{3} u / \partial x^{3}\right)=0$ on boundaries of $\Omega$, or as $h(x) \neq 0, \partial^{3} u / \partial x^{3}=0$ on boundaries of $\Omega$.

In conclusion $u$ is solution of the initial equation (41), then we enunciate the following theorem.

Theorem 12. For $u_{0} \in H^{1}(\Omega)$, exists a unique solution $u \in$ $L^{2}(0, T ; \mathscr{V}) \cap C\left([0, T] ; H^{1}(\Omega)\right)$, verifying

$$
\begin{gathered}
\frac{\partial u}{\partial t}+\frac{\partial}{\partial x}\left(h(x) \frac{\partial^{3} u}{\partial x^{3}}\right)=0 \quad \text { in }[0, T] \times \Omega, \\
\left.\frac{\partial^{3} u}{\partial x^{3}}\right|_{\partial \Omega}=0 \quad(\text { on boundaries of } \Omega), \\
\left.\frac{\partial u}{\partial n}\right|_{\partial \Omega}=0 \quad(\text { on boundaries of } \Omega), \\
u(0, x)=u_{0} \quad \text { in } \Omega .
\end{gathered}
$$

4.3.5. Some Remarks on the Modeling Space. The nullity condition of the average for functions in $\mathscr{V}$ has been decisive in the construction of the norm on this space. This space is prima facie clearly not natural for EMD because of entry function $u_{0}$ that is not necessarily zero mean. But at the cost of working with an initial condition $v_{0} \in H^{1}(\Omega)$, such that $v_{0}=$ $u_{0}-(1 /|\Omega|) \int_{\Omega} u_{0} d x$, we can consider $H$ as $H^{1}(\Omega)$ functions with null local mean.

The Nullity of the Mean Envelope: A Characteristic of $\mathscr{V}$ and EMD Algorithm. This condition, far from being superfluous, was even expected because the space $\mathscr{V}$ is the space of the mean envelopes which must be zero mean in EMD stifting process for mode extraction. If it were to calculate the upper or lower envelopes, it should be noted that the condition of the mean nullity raised a query. In EMD algorithm, this nullity condition is affected on the mean envelope and not on upper or lower envelopes. This question does not arise in the model giving the envelope interpolating inflection points of a signal. The elements of $\mathscr{V}_{e}$ are not zero mean on $\Omega$, but on a non-empty subset $\Gamma_{\Omega}$ of $\Omega$.

The nullity of the mean of the signal on a $\Gamma_{\Omega}$ is not immediately visible and warranty to decompose any function.
We show in Lemma A.1 that each relevant function to be decomposed by EMD is an integrable function possessing at least three extrema belonging to $\mathscr{V}_{e}$. The modeling space of the extrema interpolation is $\mathscr{V}_{e}$ defined in (52).

Let $f$ be a function admitting at least three extrema we can consider that $f$ passes at least once a zero at a point $x_{z}$ in its definition domain. Otherwise, we just work with the translatory (as our model is invariant by translation of the input function) vector which is equals to the half amplitude of $f$.

\section{Convergence of the Sequence $\left(u_{n}\right)_{n \in \mathbb{N}}$ of Solutions of (41) to a Solution of the Called Degenerate Problem}

In the following sections, we demonstrate that the sequence $\left(u_{n}\right)_{n \in \mathbb{N}}$ of solutions of the non degenerate problem is bounded. Next, we prove that there exists a subsequence of $\left(u_{n}\right)_{n \in \mathbb{N}}$ which converges weakly to an element $u \in \mathscr{V}$ and, finally, that this element is solution of the degenerate initial problem.

5.1. Some Estimations. From (28) we have in the distributions sense (multiplying by $\partial^{2} u_{n} / \partial x^{2}$ ):

$$
\int_{\Omega} \frac{\partial u_{n}}{\partial t} \frac{\partial^{2} u_{n}}{\partial x^{2}} d x+\int_{\Omega} \frac{\partial}{\partial x}\left(h_{n}(x) \frac{\partial^{3} u_{n}}{\partial x^{3}}\right) \frac{\partial^{2} u_{n}}{\partial x^{2}} d x=0,
$$

which is equivalent to

$$
\left\langle\frac{\partial u_{n}}{\partial t}, \frac{\partial^{2} u_{n}}{\partial x^{2}}\right\rangle_{V^{\prime} V}+\left\langle\frac{\partial}{\partial x}\left(h_{n}(x) \frac{\partial^{3} u_{n}}{\partial x^{3}}\right), \frac{\partial^{2} u_{n}}{\partial x^{2}}\right\rangle_{V^{\prime} V}=0
$$

After integration by parts, it comes out that

$$
\frac{1}{2} \int_{\Omega} \frac{\partial}{\partial t}\left(\left|\frac{\partial u_{n}}{\partial x}\right|^{2}\right) d x+\int_{\Omega} h_{n}(x)\left(\frac{\partial^{3} u_{n}}{\partial x^{3}}\right)^{2} d x=0
$$

then by integrating with respect to the variable $t \in] 0, T[$, it comes out that

$$
\begin{gathered}
\frac{1}{2}\left\|\frac{\partial u_{n}(t, \cdot)}{\partial x}\right\|_{L^{2}(\Omega)}^{2}-\frac{1}{2}\left\|\frac{\partial u_{0}}{\partial x}\right\|_{L^{2}(\Omega)}^{2} \\
\quad+\int_{0}^{t} \int_{\Omega} h_{n}(x)\left(\frac{\partial^{3} u_{n}}{\partial x^{3}}\right)^{2} d x d s=0,
\end{gathered}
$$

which implies that

$$
\begin{gathered}
\left\|\frac{\partial u_{n}(t, \cdot)}{\partial x}\right\|_{L^{2}(\Omega)}^{2}+2 \int_{0}^{t} \int_{\Omega} h_{n}(x)\left(\frac{\partial^{3} u_{n}}{\partial x^{3}}\right)^{2} d x d s \\
=\left\|\frac{\partial u_{0}}{\partial x}\right\|_{L^{2}(\Omega)}^{2} .
\end{gathered}
$$


But as $h_{n}(x) \geq \alpha$, we have

$$
\begin{aligned}
& \left\|\frac{\partial u_{n}(t, \cdot)}{\partial x}\right\|_{L^{2}(\Omega)}^{2}+2 \int_{0}^{t} \int_{\Omega} h_{n}(x)\left(\frac{\partial^{3} u_{n}}{\partial x^{3}}\right)^{2} d x d s \\
& \geq\left\|\frac{\partial u_{n}(t, \cdot)}{\partial x}\right\|_{L^{2}(\Omega)}^{2}+2 \alpha \int_{0}^{t} \int_{\Omega}\left(\frac{\partial^{3} u_{n}}{\partial x^{3}}\right)^{2} d x d s
\end{aligned}
$$

or

$$
\begin{gathered}
\left\|\frac{\partial u_{n}(t, \cdot)}{\partial x}\right\|_{L^{2}(\Omega)}^{2}+2 \int_{0}^{t} \int_{\Omega} h_{n}(x)\left(\frac{\partial^{3} u_{n}}{\partial x^{3}}\right)^{2} d x d s \\
\geq\left\|\frac{\partial u_{n}(t, \cdot)}{\partial x}\right\|_{L^{2}(\Omega)}^{2}+2 \alpha \int_{0}^{t}\left\|\frac{\partial^{3} u_{n}}{\partial x^{3}}\right\|_{L^{2}(\Omega)}^{2} d s,
\end{gathered}
$$

from which

$$
\begin{aligned}
\left\|\frac{\partial u_{0}}{\partial x}\right\|_{L^{2}(\Omega)}^{2} \geq & \left\|\frac{\partial u_{n}(t, \cdot)}{\partial x}\right\|_{L^{2}(\Omega)}^{2} \\
& +2 \alpha \int_{0}^{t}\left[\left\|u_{n}\right\|_{\mathscr{V}}^{2}-\left\|\frac{\partial u_{n}(s, \cdot)}{\partial x}\right\|_{L^{2}(\Omega)}^{2}\right] d s .
\end{aligned}
$$

Thus, on one hand,

$$
\left\|\frac{\partial u_{0}}{\partial x}\right\|_{L^{2}(\Omega)}^{2} \geq\left\|\frac{\partial u_{n}(t, \cdot)}{\partial x}\right\|_{L^{2}(\Omega)}^{2} .
$$

But

$$
\left\|\frac{\partial u_{n}(t, \cdot)}{\partial x}\right\|_{L^{2}(\Omega)}^{2} \geq \lambda^{2}\left\|u_{n}\right\|_{H^{1}(\Omega)}^{2},
$$

where $\lambda$ comes from inequality (49), which implies that

$$
\begin{aligned}
& \left\|u_{n}\right\|_{L^{\infty}\left(0, T ; H^{1}(\Omega)\right)}^{2} \leq \frac{1}{\lambda^{2}}\left\|\frac{\partial u_{0}}{\partial x}\right\|_{L^{2}(\Omega)}^{2}, \\
& \left\|u_{n}\right\|_{L^{2}\left(0, T ; H^{1}(\Omega)\right)}^{2} \leq \frac{T}{\lambda^{2}}\left\|\frac{\partial u_{0}}{\partial x}\right\|_{L^{2}(\Omega)}^{2} .
\end{aligned}
$$

Then, the sequence $\left(u_{n}\right)_{n \in \mathbb{N}}$ is bounded in $L^{\infty}\left(0, T ; H^{1}(\Omega)\right)$ and in $L^{2}\left(0, T ; H^{1}(\Omega)\right)$. And on the other hand

$$
2 \alpha \int_{0}^{T}\left[\|\| u_{n}\left\|_{\mathscr{V}}^{2}-\right\| \frac{\partial u_{n}(s, \cdot)}{\partial x} \|_{L^{2}(\Omega)}^{2}\right] d s \leq\left\|\frac{\partial u_{0}}{\partial x}\right\|_{L^{2}(\Omega)}^{2},
$$

which gives

$$
\int_{0}^{T} \mid\left\|u_{n}\right\|_{\mathscr{V}}^{2} d s \leq \int_{0}^{T}\left\|\frac{\partial u_{n}(s, \cdot)}{\partial x}\right\|_{L^{2}(\Omega)}^{2} d s+\frac{1}{2 \alpha}\left\|\frac{\partial u_{0}}{\partial x}\right\|_{L^{2}(\Omega)}^{2} .
$$

But as

$$
\left\|\frac{\partial u_{n}(s, \cdot)}{\partial x}\right\|_{L^{2}(\Omega)}^{2} \leq\left\|u_{n}(s, \cdot)\right\|_{H^{1}(\Omega)}^{2}
$$

therefore

$$
\int_{0}^{T} \mid\left\|u_{n}\right\|_{\mathscr{V}}^{2} d s \leq \int_{0}^{T}\left\|u_{n}(s, \cdot)\right\|_{H^{1}(\Omega)}^{2} d s+\frac{1}{2 \alpha}\left\|\frac{\partial u_{0}}{\partial x}\right\|_{L^{2}(\Omega)}^{2}
$$

Thus,

$$
\int_{0}^{T}\left|\left\|u_{n}\right\|\right|_{\mathscr{V}}^{2} d s \leq T\left\|u_{n}\right\|_{L^{\infty}\left(0, T ; H^{1}(\Omega)\right)}^{2}+\frac{1}{2 \alpha}\left\|\frac{\partial u_{0}}{\partial x}\right\|_{L^{2}(\Omega)}^{2},
$$

which implies according to (84) that

$$
\int_{0}^{T} \mid\left\|u_{n}\right\|_{\mathscr{V}}^{2} d s \leq \frac{T}{\lambda^{2}}\left\|\frac{\partial u_{0}}{\partial x}\right\|_{L^{2}(\Omega)}^{2}+\frac{1}{2 \alpha}\left\|\frac{\partial u_{0}}{\partial x}\right\|_{L^{2}(\Omega)}^{2}
$$

We finally deduced the second estimate

$$
\begin{aligned}
\left\|u_{n}\right\|_{L^{2}(0, T ; \mathscr{V})}^{2} & =\left\|u_{n}\right\|_{L^{2}\left(0, T ; \mathscr{V}_{e}\right)}^{2} \\
& \leq\left(\frac{T}{\lambda^{2}}+\frac{1}{2 \alpha}\right)\left\|\frac{\partial u_{0}}{\partial x}\right\|_{L^{2}(\Omega)}^{2}
\end{aligned}
$$

Then, the sequence $\left(u_{n}\right)_{n \in \mathbb{N}}$ is bounded in $L^{2}(0, T ; \mathscr{V})$.

5.2. Weak Convergence of the Subsequence of $\left(u_{n}\right)_{n \in \mathbb{N}}$. The sequence $\left(u_{n}\right)_{n \in \mathbb{N}}$ is bounded in the space $L^{2}(0, T ; \mathscr{V})$. Then there exists a subsequence $\left(u_{n_{k}}\right)_{k \in \mathbb{N}}$ that converge weakly to $u$ in $L^{2}(0, T ; \mathscr{V})$ with $d u / d t \in L^{2}\left(0, T ; V^{\prime}\right)$.

Leting $v \in \mathscr{V}$ be a test function, we have the following.

Firstly. As $u_{n_{k}} \rightarrow u$ weakly in $L^{2}(0, T ; V)$, therefore, $u_{n_{k}} \rightarrow u$ weakly in $L^{2}\left(0, T ; H^{1}(\Omega)\right)$.

Thus, in the distributions sense,

$$
\int_{\Omega} \frac{d u_{n_{k}}}{d t} \frac{\partial^{2} v}{\partial x^{2}} d x=\frac{d}{d t} \int_{\Omega} \frac{\partial u_{n_{k}}}{\partial x} \frac{\partial v}{\partial x} d x
$$

by integrating on $[0, t]$, we have

$$
\begin{aligned}
\int_{0}^{t} \int_{\Omega} \frac{d u_{n_{k}}}{d s} \frac{\partial^{2} v}{\partial x^{2}} d x d s & =-\int_{0}^{t} \frac{d}{d s} \int_{\Omega} \frac{\partial u_{n_{k}}}{\partial x} \frac{\partial v}{\partial x} d x d s \\
& =-\int_{0}^{t}\left\langle\frac{d u_{n_{k}}}{d s}, \frac{\partial^{2} v}{\partial x^{2}}\right\rangle_{V^{\prime} V} d s \\
& =-\int_{\Omega}\left(u_{n_{k}}(t)-u_{n_{k}}(0)\right) \frac{\partial^{2} v}{\partial x^{2}} d x
\end{aligned}
$$

By integrating by parts again the last equation, we have

$$
\begin{aligned}
\int_{\Omega}\left(u_{n_{k}}(t)-u_{n_{k}}(0)\right) \frac{\partial^{2} v}{\partial x^{2}} d x= & \int_{\Omega} \frac{\partial u_{n_{k}}(t)}{\partial x} \frac{\partial v}{\partial x} d x \\
& -\int_{\Omega} \frac{\partial u_{n_{k}}(0)}{\partial x} \frac{\partial v}{\partial x} d x
\end{aligned}
$$


This expression converges to

$$
\begin{aligned}
\int_{\Omega} \frac{\partial u(t)}{\partial x} \frac{\partial v}{\partial x} d x-\int_{\Omega} \frac{\partial u_{0}}{\partial x} \frac{\partial v}{\partial x} d x & =\int_{\Omega}\left[\frac{\partial u(t)}{\partial x}-\frac{\partial u_{0}}{\partial x}\right] \frac{\partial v}{\partial x} d x \\
& =\int_{\Omega}\left[u(t)-u_{0}\right] \frac{\partial^{2} v}{\partial x^{2}} d x \\
& =\int_{0}^{t}\left\langle\frac{d u}{d s}, \frac{\partial^{2} v}{\partial x^{2}}\right\rangle_{V^{\prime} V} d s
\end{aligned}
$$

Therefore, $\int_{0}^{t} \int_{\Omega}\left(d u_{n_{k}} / d s\right)\left(\partial^{2} v / \partial x^{2}\right) d x d s$ converges when $n_{k}$ tends to infinity to

$$
\int_{0}^{t} \int_{\Omega} \frac{d u}{d s} \frac{\partial^{2} v}{\partial x^{2}} d x d s
$$

Consequently, $\partial u_{n_{k}} / \partial t \rightarrow \partial u / \partial t$ weakly in $L^{2}\left(0, T ; \mathscr{V}^{\prime}\right)$.

Secondly. As $h_{n}(x)=h(x)+(1 / n)$,

$$
\begin{aligned}
& \text { we have } \frac{\partial}{\partial x}\left(h_{n_{k}} \frac{\partial^{3} u_{n_{k}}}{\partial x^{3}}-h(x) \frac{\partial^{3} u}{\partial x^{3}}\right) \\
& =\frac{\partial}{\partial x}\left(h(x) \frac{\partial^{3}}{\partial x^{3}}\left(u_{n_{k}}-u\right)\right)+\frac{1}{n_{k}} \frac{\partial^{4} u_{n_{k}}}{\partial x^{4}} .
\end{aligned}
$$

However, $\int_{\Omega} \frac{\partial^{4} u_{n_{k}}}{\partial x^{4}} v d x=\int_{\partial \Omega} \frac{\partial^{3} u}{\partial x^{3}} v \cdot n d \sigma$

$$
-\int_{\Omega} \frac{\partial v}{\partial x} \frac{\partial^{3} u_{n_{k}}}{\partial x^{3}} d x
$$

And because $\left.\frac{\partial^{3} u}{\partial x^{3}}\right|_{\partial \Omega}=0, \quad \frac{\partial^{3} u_{n_{k}}}{\partial x^{3}}$ converges to $\frac{\partial^{3} u}{\partial x^{3}}$

$$
\text { in } L^{2}\left(0, T ; L^{2}(\Omega)\right) \text {, }
$$

then,

$$
\begin{aligned}
\frac{1}{n_{k}}\left|\int_{\Omega} \frac{\partial^{4} u_{n_{k}}}{\partial x^{4}} v d x\right| & =\frac{1}{n_{k}}\left|\int_{\Omega} \frac{\partial^{3} u_{n_{k}}}{\partial x^{3}} \frac{\partial v}{\partial x} d x\right| \\
& \leq \frac{1}{n_{k}}\left\|\frac{\partial v}{\partial x}\right\|_{L^{2}(\Omega)}\left\|\frac{\partial^{3} u_{n_{k}}}{\partial x^{3}}\right\|_{L^{2}(\Omega)}
\end{aligned}
$$

converges to zero when $n_{k}$ tends to infinity.

On the other hand, from (98), we have

$$
\begin{gathered}
\left|\int_{\Omega} \frac{\partial}{\partial x}\left(h(x) \frac{\partial^{3}}{\partial x^{3}}\left(u_{n_{k}}-u\right)\right) v d x\right| \\
\leq \beta\left|\int_{\Omega} \frac{\partial^{3}}{\partial x^{3}}\left(u_{n_{k}}-u\right) \frac{\partial v}{\partial x} d x\right|
\end{gathered}
$$

which converges to zero in $L^{2}\left(0, T ; L^{2}(\Omega)\right)$ according to weak convergence (see Definition 3 ) in $H^{3}(\Omega)$ of $\partial^{3} u_{n_{k}} / \partial x^{3}$ to $\partial^{3} u / \partial x^{3}$. Thus, we have shown that in the distributions sense of the one hand

$$
\int_{\Omega} \frac{d u_{n_{k}}}{d t} \frac{\partial^{2} v}{\partial x^{2}} d x
$$

converges to

$$
\int_{\Omega} \frac{d u}{d t} \frac{\partial^{2} v}{\partial x^{2}} d x
$$

and on the other hand,

$$
\int_{\Omega} \frac{\partial}{\partial x}\left(h_{n_{k}} \frac{\partial^{3} u_{n_{k}}}{\partial x^{3}}\right) v d x
$$

converges to

$$
\int_{\Omega} \frac{\partial}{\partial x}\left(h(x) \frac{\partial^{3} u}{\partial x^{3}}\right) v d x
$$

Consequently, for each $v \in L^{2}\left(0, T ; V^{\prime}\right)$,

$$
\int_{\Omega} \frac{d u_{n_{k}}}{d t} v d x+\int_{\Omega} \frac{\partial}{\partial x}\left(h_{n_{k}} \frac{\partial^{3} u_{n_{k}}}{\partial x^{3}}\right) v d x
$$

converges in the distributions sense to

$$
\int_{\Omega} \frac{d u}{d t} v d x+\int_{\Omega} \frac{\partial}{\partial x}\left(h(x) \frac{\partial^{3} u}{\partial x^{3}}\right) v d x
$$

that is to say, $\left\langle\left(d u_{n_{k}} / d t\right), v\right\rangle_{V^{\prime} V}+\left\langle(\partial / \partial x)\left(h_{n_{k}}(x)\left(\partial^{3} u_{n_{k}} / \partial x^{3}\right)\right)\right.$, $v\rangle_{V^{\prime} V}$ converges when $n_{k}$ tends to infinity to $\langle(d u / d t), v\rangle_{V^{\prime} V^{+}}$ $\left\langle(\partial / \partial x)\left(h(x)\left(\partial^{3} u / \partial x^{3}\right)\right), v\right\rangle_{V^{\prime} V}$. It remains to show that the limit $u$ is a solution of the degenerate problem. But $\int_{\Omega}\left(d u_{n_{k}} / d t\right) v d x+\int_{\Omega}(\partial / \partial x)\left(h_{n_{k}}\left(\partial^{3} u_{n_{k}} / \partial x^{3}\right)\right) v d x=0$, which implies in the distributions sense $\int_{\Omega}(d u / d t) v d x+$ $\int_{\Omega}(\partial / \partial x)\left(h(x)\left(\partial^{3} u / \partial x^{3}\right)\right) v d x=0$. In other words, the weak limit of the sequence $\left(u_{n_{k}}\right)_{k \in \mathbb{N}}$ is weak solution of the initial degenerate problem. Moreover, this solution is unique and is in $L^{2}(0, T ; \mathscr{V}) \cap C\left([0, T] ; H^{1}(\Omega)\right)$. Better, using the compact inclusion of $\mathscr{V}$ (which behaves like $H^{3}(\Omega)$ because of the equivalence of the norms) in $H^{2}(\Omega)$, we conclude that the sequence of solutions of non-degenerate problem (approximated problem) converges strongly in $H^{2}(\Omega)$ to the solution of the initial degenerate problem. The space $H^{2}(\Omega)$ is, moreover, the space of strong solutions of the initial system (25). 


\section{Numerical Implementation and Applications}

Deliberately, we present the numerical implementation in the case of dimension $n=2$. The reason is that we give examples both on $1 \mathrm{D}$ and $2 \mathrm{D}$ signals.

In the sequel, we adopt the following notation. The mesh of the domain $\Omega \times[0, T]$ is given by

$$
\left(t_{k}, x_{i}\right)=(k \Delta t, i \Delta x) \text { for } k \geq 0, i \in \mathbb{N} \text {. }
$$

We note by $U_{i}^{k}$ the approximate value of the solution $u(t, x)$ at the point $(k \Delta t, i \Delta x)=(k \Delta t, i)$.

The discrete solution at the iteration $k$ is obtained at the discrete time $t_{k}$ and is denoted by $U^{k}$. An approximation of the temporal derivative is $u_{t}=\partial u / \partial t$ at this same time $t_{k}=$ $k \Delta t$ is then $\left(U^{k+1}-U^{k}\right) / \Delta t$.

6.1. Numerical Schemes for the PDE Resolution. The discrete model calculating the mean envelope can be written as follows:

$$
u_{t}=-\sum_{i, j=1}^{2} \partial_{x_{i}}^{1}\left(g_{i, j} \partial_{x_{i}}^{1} \partial_{x_{j}}^{2} u\right)
$$

where $\partial_{x_{i}}^{q}$ denotes the derivative of order $q \in\{1,2\}$ relatively to the spatial coordinate $x_{i}$.

To take into account the discontinuity of the diffusivity function, we use the harmonic mean of $g_{i, j}$ defined by

$$
\begin{aligned}
& g_{i+1 / 2, j}=\left[\frac{g_{i, j}^{-1}+g_{i+1, j}^{-1}}{2}\right]^{-1}, \\
& g_{i-1 / 2, j}=\left[\frac{g_{i-1, j}^{-1}+g_{i+1, j}^{-1}}{2}\right]^{-1}, \\
& g_{i, j+1 / 2}=\left[\frac{g_{i, j}^{-1}+g_{i, j+1}^{-1}}{2}\right]^{-1}, \\
& g_{i, j-1 / 2}=\left[\frac{g_{i, j}^{-1}+g_{i, j-1}^{-1}}{2}\right]^{-1} .
\end{aligned}
$$

Recall that $g$ is strictly positive, which allows its inversion in the previous system.
We define also the numerical derivatives of $v$ by

$$
D_{i}^{+} v(i, j)=v(i+1, j)-v(i, j)
$$

forward difference on the $x_{i}$-direction,

$$
D_{i}^{-} v(i, j)=v(i, j)-v(i-1, j)
$$

backward difference on the $x_{i}$-direction,

$$
D_{i}^{0} v(i, j)=v\left(i+\frac{1}{2}, j\right)-v\left(i-\frac{1}{2}, j\right)
$$

central difference on the $x_{i}$-direction,

$$
D_{j}^{+} v(i, j)=v(i, j+1)-v(i, j)
$$

forward difference on the $x_{j}$-direction,

$$
D_{j}^{-} v(i, j)=v(i, j-1)-v(i, j)
$$

backward difference on the $x_{j}$-direction,

and finally we pose $w_{i j} u(i, j)=D_{i}^{+} D_{j}^{-} D_{j}^{+} u(i, j)$. For positive integer, $i$ and $j$, the numerical approximation $L_{i j} u$, of $g_{i, j} \partial_{x_{i}}^{1} \partial_{x_{j}}^{2} u$ in (108) is given by

$$
\begin{aligned}
L_{i j}= & g_{i+1 / 2, j} D_{i}^{+} w_{i j}-g_{i-1 / 2, j} D_{i}^{-} w_{i j} \\
& +g_{i, j+1 / 2} D_{j}^{+} w_{j i}-g_{i, j-1 / 2} D_{j}^{-} w_{j i} .
\end{aligned}
$$

We present the first natural explicit scheme.

6.1.1. An Explicit Scheme. An explicit scheme to solve the PDE is the following: $\left(U^{k+1}-U^{k}\right) / \Delta t=-\sum_{i, j=1}^{2} L_{i j} U^{k}$ or $U^{k+1}=\left(I-\Delta t \sum_{i, j=1}^{2} L_{i j}\right) U^{k}$. Let us pose $\mathfrak{D}=I-\Delta t \sum_{i, j=1}^{2} L_{i j}$.

Thus,

$$
U^{k+1}=\mathfrak{D} U^{k} \quad \text { with } U^{0}=u_{0} .
$$

If $N$ is the image or the signal size, the matrix $\mathscr{D}$ is a sparse matrix of order $N^{2}$.

However, this explicit scheme requires for its stability $\Delta t$ very small. To overcome this drawback, other numerical schemes can be used such as Du Fort and Frankel [22], which is unconditionally stable, but conditionally consisting of time steps $\Delta t$ not too great, to satisfy the condition of numerical stability of Courant-Friedrich-Levy [26] schemes. Another very effective scheme is proposed by Vogel and Oman in [27] it is based on the principle of the fixed point. It allows us to address the problem stationary with the result of the existence of the asymptotic solution. With this scheme, the solution is an eigenvector of the operator represented by the matrix $\mathscr{D}$.

6.1.2. The Additive Operator Splitting Scheme. We can use the additive operator splitting scheme (AOS) which is written as follows:

$$
U^{k+1}=\frac{1}{2} \sum_{p=1}^{2}\left(I+2 \Delta t L_{p p}\right)^{-1}\left(I-\Delta t \sum_{i=1}^{2} \sum_{j \neq i} L_{i j}\right) U^{k} .
$$


This scheme is more stable, but requires a matrix inversion, even if the matrix is pentadiagonal. This inversion may be affected by the Thomas [28] algorithm modified. In (113), the reverse of the matrix is independent of the iteration $k$ and is carried out once only for $k=0$.

6.1.3. The Alternate Direction Implicit Scheme (ADI). Simplification which reduces the complexity of the computation of the solution can be made in the implementation of the numerical equation (108) combining an implicit form for half the computation time and an explicit form for the remainder.

It is more difficult to implement this scheme, ADI, for the fourth-order PDE. This is due to cross derivatives in (108). Witelski and Bowen [29] suggest a readjustment of the ADI in which a mixed derivative is calculated explicitly in (108) by the following:

$$
U^{k+1}=\left(\prod_{p=1}^{2}\left(I-\Delta t L_{p p}\right)\right)^{-1}\left(I+\Delta t \sum_{i=1}^{2} \sum_{j \neq i} L_{i j}\right) U^{k} .
$$

Besides the approximation errors inherent in all numerical scheme, the fundamental difference between theses methods remains the computation time.

6.2. The Boundary Conditions. The boundary conditions used are those of system (41). In fact, the solution is a polynomial of order 3 and can be written as $u(x)=a_{3} x^{3}+$ $a_{2} x^{2}+a_{1} x+a_{0}$. The nullity of its derivatives of orders 3 and 1 on the edges leads to $a_{3}=0, \partial u / \partial x=2 a_{2} x+a_{1}=0$ on the boundary. Hence, $a_{2}=a_{1}=0$, which finally gives $u(x)=$ $a_{0}$. We have taken as boundary conditions that outside, the domain, the signal is constant. This means into practice the cancellation of all the derivatives of order less than or equal to 3. For diffusivity function at the edges, it is constant. When this constant is null, we join a nonnatural manner to care of edge effects, which was to take the signal ends at the same time as local maximum and local minimum. We do not this here.

6.3. Test Results of PDE Interpolator on Signal. In our numerical tests, we used the implicit scheme of Crank-Nikelson which gives good results with $\Delta t$ that can take very large values. In the following, we give some examples of calculation of upper and lower envelopes and for the mean envelope interpolating the inflection points of the signal. This scheme in consistent of order 1 both in space and in time.

6.3.1. Some Examples. First, we present in Figure 1 a pathological case of a signal without local extremum, when we cannot calculate the envelopes by spline interpolation of the extrema. Our PDE interpolator works well with the max-min curvature points detection in the diffusivity function.

Figure 2 represents the evolution of the PDE solution with the form given by (14). Upon iteration 40000, the solution almost evolves addition; it is potentially an envelope.

In Figure 3, we represent the envelope calculation of the same function with the form given by (16) for different

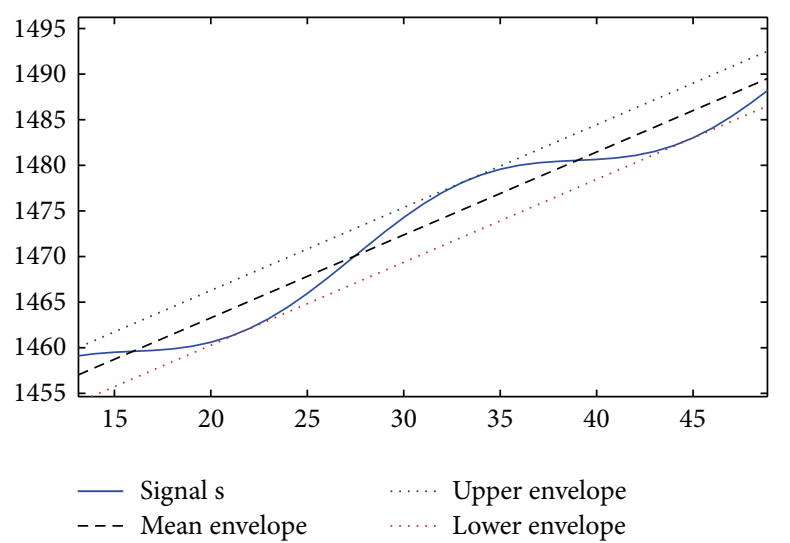

FIgURE 1: PDE interpolator for signal without local extrema. The input signal is in blue unbroken line, and the envelopes are in dotted lines.

tension factor $\theta$. We can see that the regularity of envelopes is no longer for values of $\theta$ close to zero.

In Figure 4, we represent the envelope calculation with the form given by (17) for different tension factor $\theta=0$.

In Figure 5, we make a comparison between our PDE interpolator and the cubic spline interpolation for an input signal $s$ that equal to the superposition of two sinusoidal components with different amplitudes and frequencies, $s_{1}$ and $s_{1}$. When it comes to interpolate the classical local extrema, we have almost the same result, the two not being satisfactory, which certainly requires a bit more iterations in the sifting process. In contrast by, our interpolator which passes through the points of maximum and minimum local curvature gives the correct envelopes quite properly.

\subsubsection{The Specific Case of the PDE Interpolator of the Turning} Points. In the case of the PDE interpolator of inflection points, we have the advantage of not only calculating asymptotic solution of PDE but also dividing the computing time almost in half. Only, we must numerically have a good estimate of the envelope to guard against a sampling of the input signal which must also be quite dense. This model asks more numerically regularity and in some cases an oversampling.

In Figures 6 and 7, we proceed to calculate the solution of the PDE interpolator passing through the inflection points, for different sampling for the same composite signal $s=$ $s_{1}+s_{2}$. The convergence is slower if the number of sampling points is higher, but the solution is a good estimate of the mean envelope. In Figure 6, with a more dense sampling, convergence is slower, and we get a mean envelope judged more suitable than 128 sampling points. In Figure 7, with adequate sampling, we presented the mean envelope expected trend, that is the component $s_{1}$ of the signal $s$. To capture the inflection points, a sampling of four once of the smallest local period of the signal is generally eligible for a suitable to estimate the envelope. This is a consequence of the theory of Shannon sampling.

6.3.3. Test on $2 D$ Signal. Finally, in Figure 8, we use the $2 D$ version of the PDE interpolator for image restoration. The 


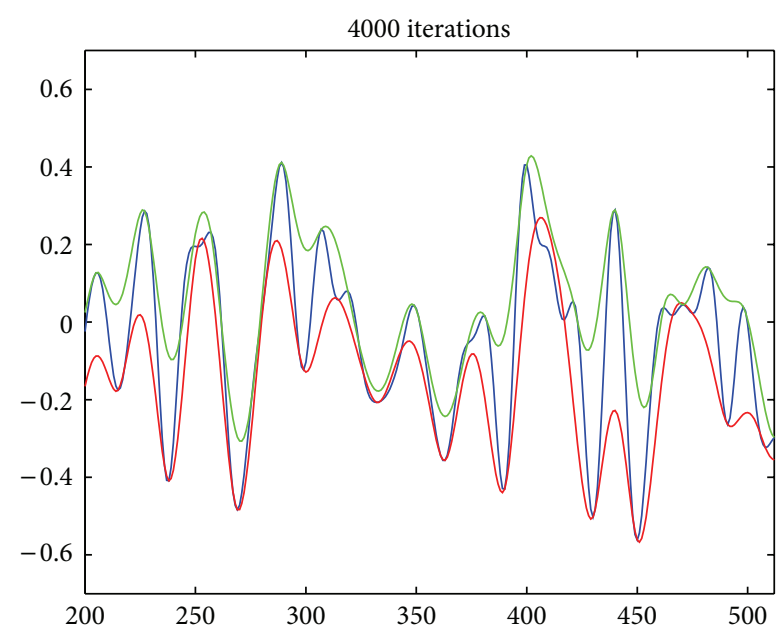

(a) Input signal is in blue color. In red and green colors are the envelopes upon iteration 4000

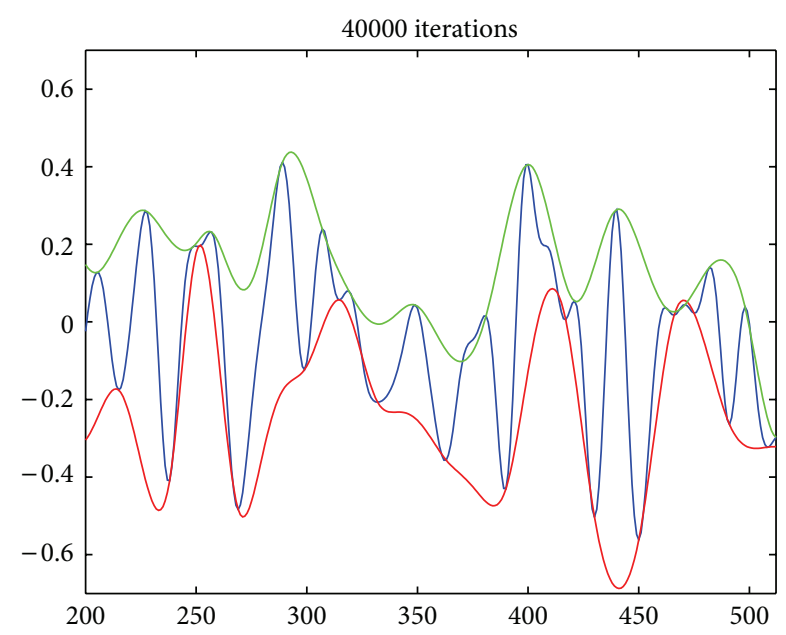

(b) Input signal is in blue color. In red and green colors are the envelopes upon iteration 40000

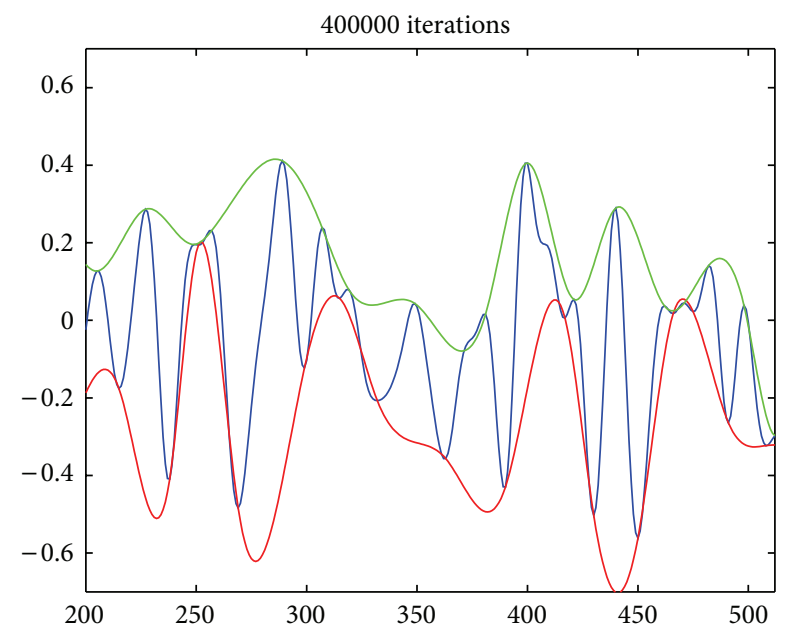

(c) Input signal is in blue color. In red and green colors are the envelopes upon iteration 400000

FIGURE 2: In (a) input signal is in blue color. In red and green colors are the envelopes calculus upon iteration 4000. In (b) the envelopes calculus upon iteration 40000. In (c) the envelopes calculus upon iteration 400000.

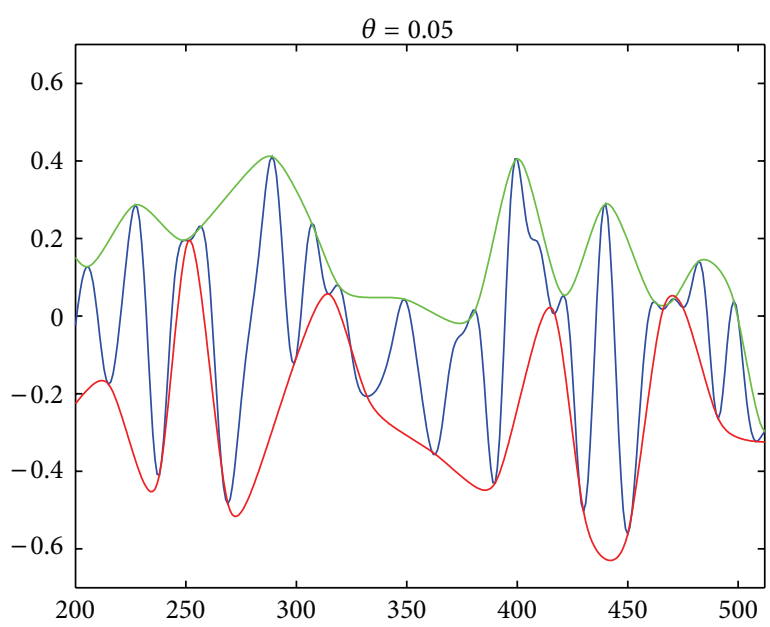

(a) Input signal is in blue color. In red and green colors are the envelopes at the convergence for $\theta=0.05$

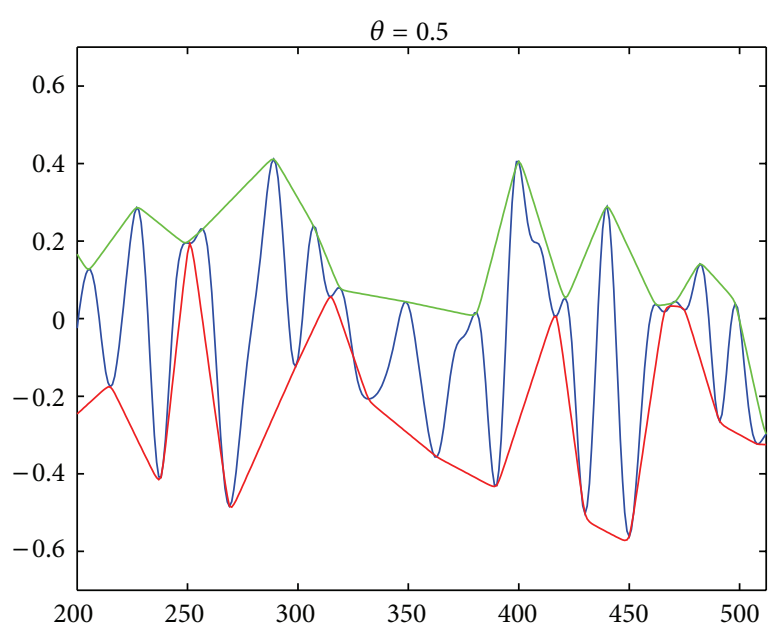

(b) Input signal is in blue color. In red and green colors are the envelopes at the convergence for $\theta=0.5$

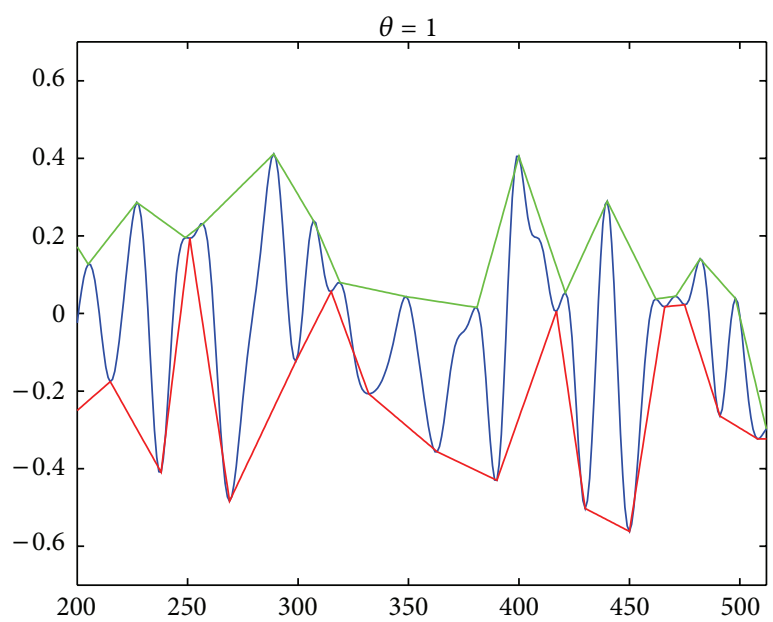

(c) Input signal is in blue color. In red and green colors are the envelopes at the convergence for $\theta=1$

FIgURE 3: In ( $a, b$, and $\mathrm{c}$ ) input signal is in blue color. In red and green colors are the envelopes at the convergence of the evolutive PDE. 


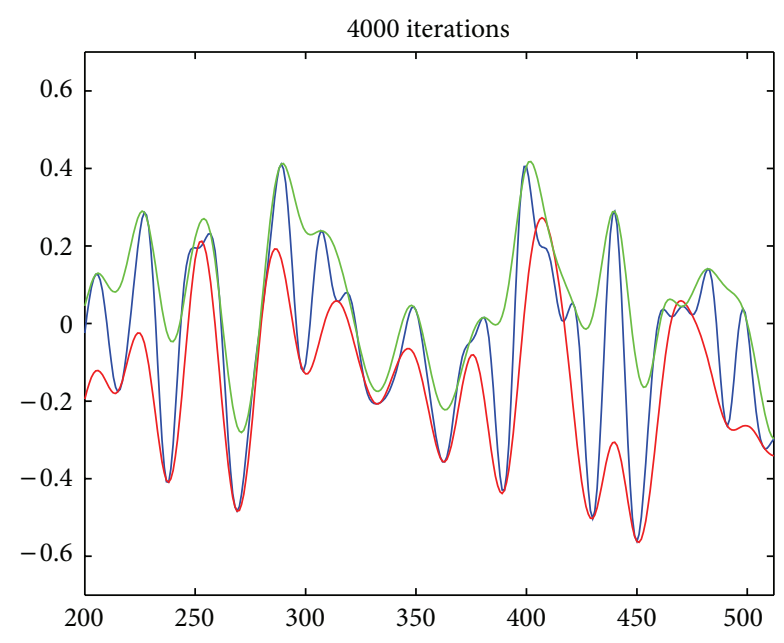

(a) Input signal in blue color. In red and green colors the envelopes upon iteration 4000

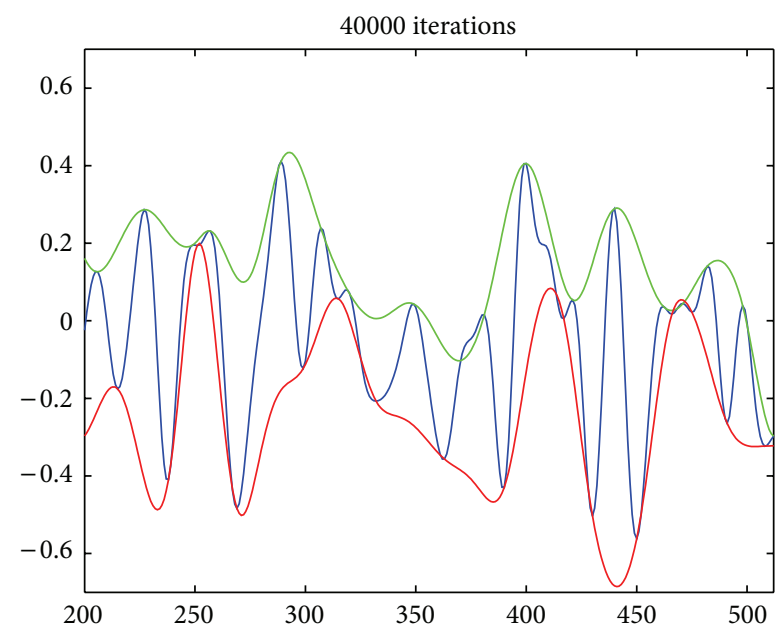

(b) Input signal in blue color. In red and green colors the envelopes upon iteration 40000

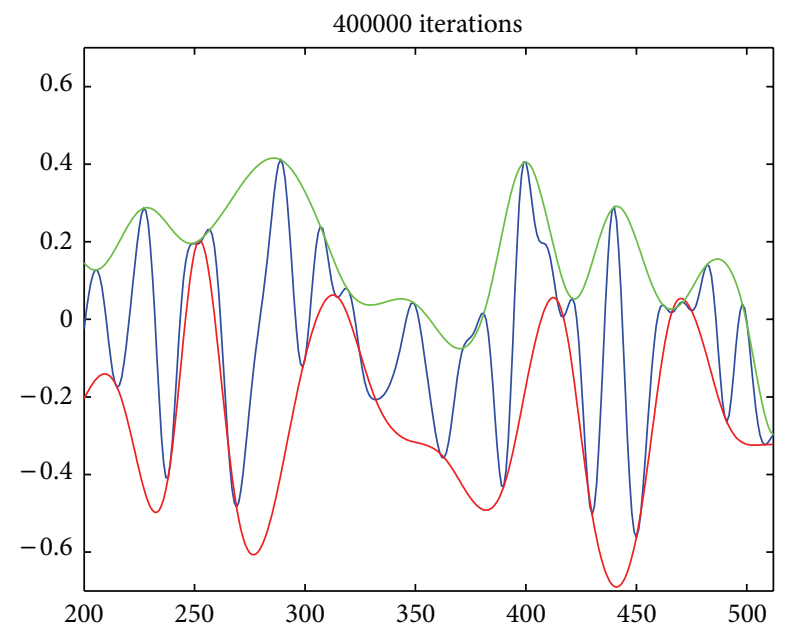

(c) Input signal in blue color. In red and green colors the envelopes upon iteration 400000

Figure 4: Envelope calculus by (17) with $\theta=0$. In (a), at this stage, the two envelopes overlap by location. In (b), less overlap and practically the asymptotic solution are almost reached. In (c), the stability of the evolution leading to the solution. corrupted image taken as an input 2D signal in our model which proceeds by edge propagating provides an interpolated version of the data that is clean enough.

\section{Conclusion}

In this paper, we model a new PDE interpolator which permits to calculate the envelopes of a signal. The existence and uniqueness of the solution to the mathematical problem are determined by a variational approach. This theoretical framework contributes to the mathematical modeling of EMD algorithm. We have shown, in particular, how the $\mathscr{V}$ space reminds the extraction conditions of the EMD modes, and we prove that the set of eligible functions to be decomposed by EMD are in $H^{1}(\Omega)$. The PDE interpolator is not based on any prior knowledge on the noise level as opposed to the total variation method in [11]. The tests in both signal and image processing demonstrate the effectiveness of the new interpolator and provide an insight into opportunities in multiscale analysis of multidimensional signal.

\section{Appendix}

Lemma A.1. Let $f \in L^{1}(\Omega)$ having at least three local extrema and null at least at one point of $\Omega$. One can assume that $f$ vanishes by changing sign. Then, there exist $\delta>0$ and $x_{c} \in \Omega$ such that the local mean at $x_{c}$ is null, for example,

$$
\operatorname{Mloc}_{\delta}[f]\left(x_{c}\right)=\frac{1}{2 \delta} \int_{x_{c}-\delta}^{x_{c}+\delta} f(s) d s=0 .
$$

Proof. Let $x_{c}$ and $\epsilon>0$ such that $f\left(x_{c}\right)=0, f\left(x_{c}-\epsilon\right)<$ 0 , and $f\left(x_{c}+\epsilon\right)>0$. Proceed by contradiction as the local mean is continuous, and $\operatorname{Mloc}_{\delta}[f]$ not null implies that it keeps a constant sign on $\Omega$. Suppose that $\forall \delta, x>$ $0, M \operatorname{loc}_{\delta}[f](x)>0$. In particular, the positive sequence $\left(\operatorname{Mloc}_{1 / n}[f]\left(x_{c}-\epsilon\right)\right)_{n \in \mathbb{N}^{*}}$ converges to $f\left(x_{c}-\epsilon\right) \geq 0$. This is absurd because $f\left(x_{c}-\epsilon\right)<0$. Thus, the proof of Lemma A.1 is performed.

From this lemma, any eligible function for EMD is in $\mathscr{V}_{e}$.

Lemma A.2. There exist two constants $C_{V}, C_{H}>0$ such that $\forall v \in \mathscr{V}:$

$$
C_{V}\|v\|_{H^{3}(\Omega)}^{2} \leq \mid\|v\|_{\mathscr{V}}^{2} \leq C_{H}\|v\|_{H^{3}(\Omega)}^{2} .
$$

\section{Proof of Lemma A.2}

Step 1 (second part of (A.2)). Rewriting first the norms, $\|v\|_{H^{3}(\Omega)}^{2}=\sum_{s=0}^{3}\left\|\partial^{s} v / \partial x^{s}\right\|_{L_{(\Omega)}^{2}}^{2}$, that is to say,

$$
\begin{aligned}
\|v\|_{H^{3}(\Omega)}^{2}= & \|v\|_{L^{2}(\Omega)}^{2}+\left\|\frac{\partial v}{\partial x}\right\|_{L^{2}(\Omega)}^{2} \\
& +\left\|\frac{\partial^{2} v}{\partial x^{2}}\right\|_{L^{2}(\Omega)}^{2}+\left\|\frac{\partial^{3} v}{\partial x^{3}}\right\|_{L^{2}(\Omega)}^{2},
\end{aligned}
$$



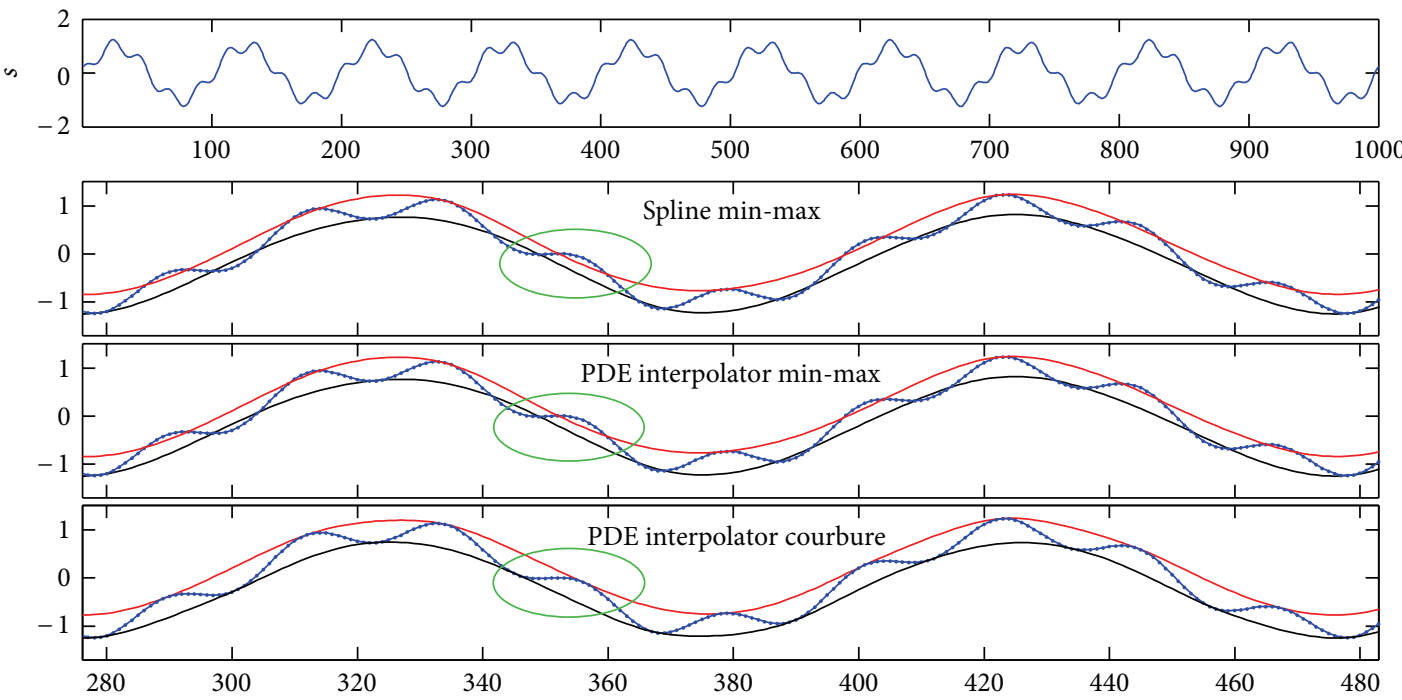

- Env. inf.

_- Env. sup.

FIgURE 5: Comparison between PDE interpolator and cubic spline. The input signal is in blue color, and in red and black colors are the envelopes.

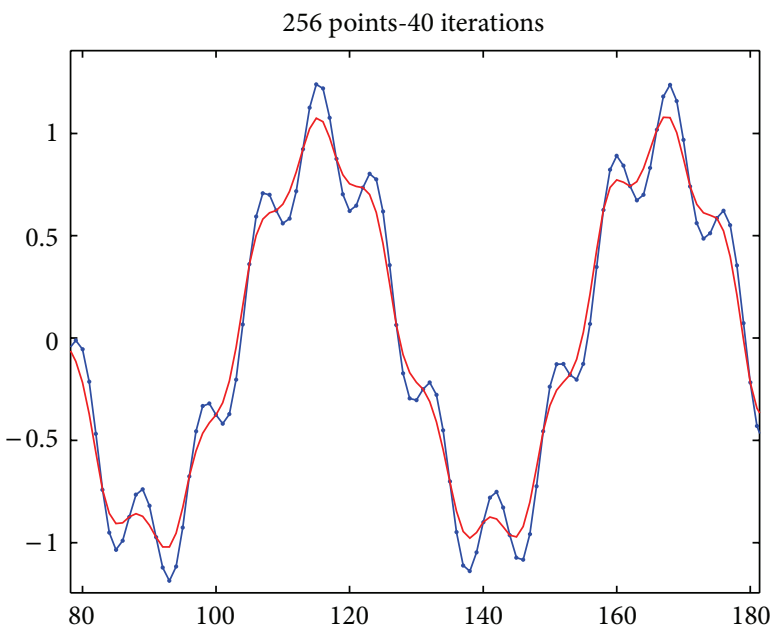

(a) Envelope after 40 iterations

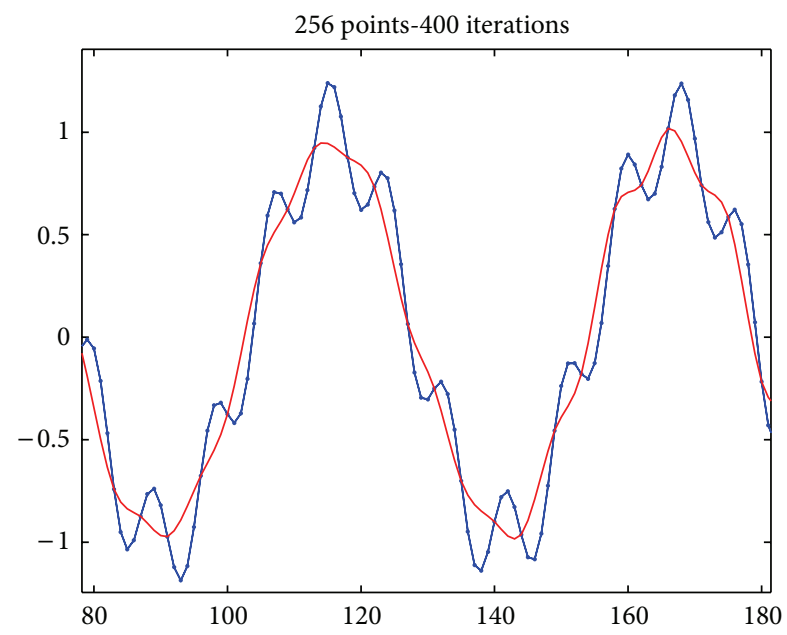

(b) Envelope after 400 iterations

Figure 6: The envelope of the signal test $s=s_{1}+s_{2}$ sampling with 256 points.

and secondly,

$$
\mid\|v\|_{\mathscr{V}}^{2}=\left\|\frac{\partial v}{\partial x}\right\|_{L^{2}(\Omega)}^{2}+\left\|\frac{\partial^{3} v}{\partial x^{3}}\right\|_{L^{2}(\Omega)}^{2},
$$

we have immediately $|\|v\||_{\mathscr{V}}^{2} \leq\|v\|_{H^{3}(\Omega)}^{2}$ posing $C_{H}=1$.

Step 2 (first part of (A.2)). Firstly,

$$
\left\|\frac{\partial^{2} v}{\partial x^{2}}\right\|_{L^{2}(\Omega)}^{2}=\int_{\Omega}\left(\frac{\partial^{2} v}{\partial x^{2}}\right)^{2} d x
$$

and by integration by parts,

$$
\int_{\Omega}\left(\frac{\partial^{2} v}{\partial x^{2}}\right)^{2} d x=\int_{\partial \Omega} \frac{\partial v}{\partial n} \frac{\partial^{2} v}{\partial x^{2}} d \sigma-\int_{\Omega} \frac{\partial v}{\partial x} \frac{\partial^{3} v}{\partial x^{3}} d x
$$

$$
\int_{\Omega}\left(\frac{\partial^{2} v}{\partial x^{2}}\right)^{2} d x=-\int_{\Omega} \frac{\partial v}{\partial x} \frac{\partial^{3} v}{\partial x^{3}} d x
$$




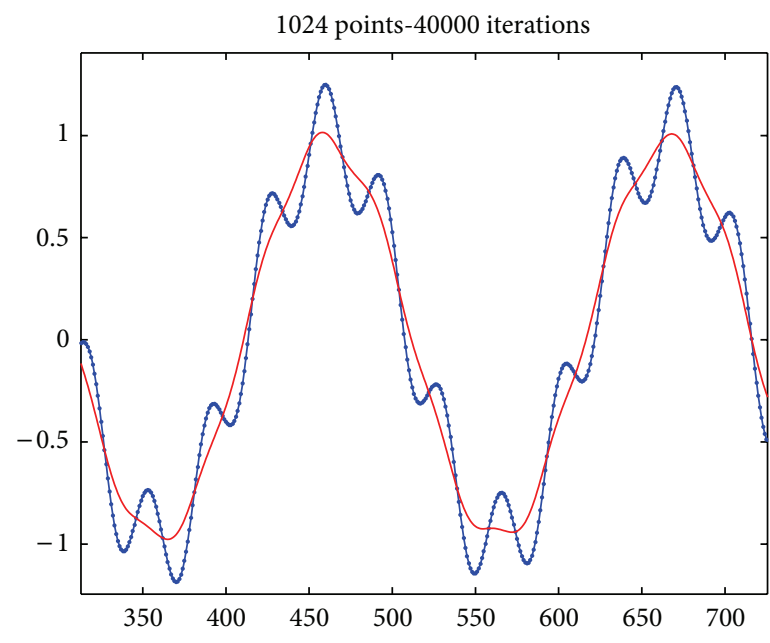

(a) Envelope after 40000 iterations

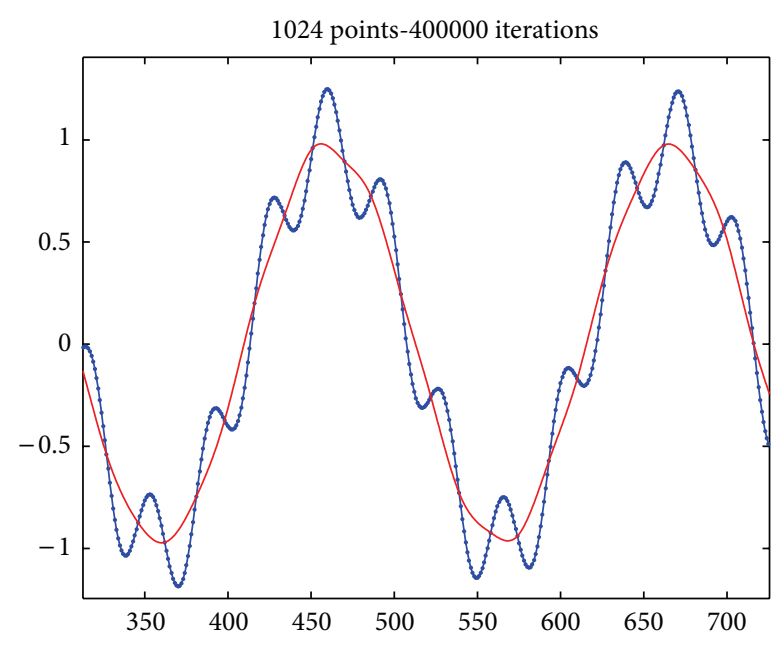

(b) Envelope after 400000 iterations

FIgURE 7: The envelope of the signal test $s=s_{1}+s_{2}$ sampling with 1024 points.

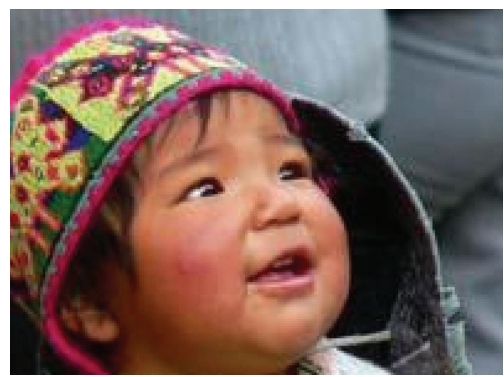

(a)

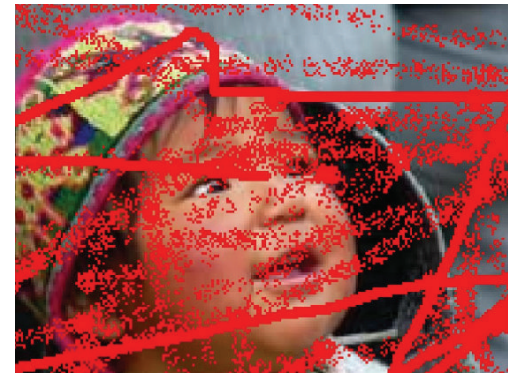

(b)

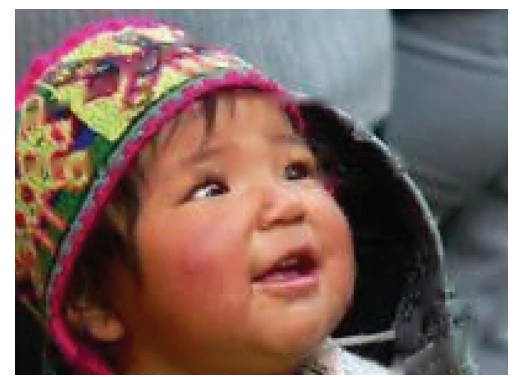

(c)

FIGURE 8: PDE interpolator for image restoration. An original image (a) is corrupted (b) and restored (c) by our 2D PDE-interpolator. Original image.

and, therefore, the use of Schwarz inegality gives

$$
\left\|\frac{\partial^{2} v}{\partial x^{2}}\right\|_{L^{2}(\Omega)}^{2} \leq\left\|\frac{\partial v}{\partial x}\right\|_{L^{2}(\Omega)}\left\|\frac{\partial^{3} v}{\partial x^{3}}\right\|_{L^{2}(\Omega)} ;
$$

then, we deduce

$$
\left\|\frac{\partial^{2} v}{\partial x^{2}}\right\|_{L^{2}(\Omega)}^{2} \leq \frac{1}{2}\left\|\frac{\partial v}{\partial x}\right\|_{L^{2}(\Omega)}^{2}+\frac{1}{2}\left\|\frac{\partial^{3} v}{\partial x^{3}}\right\|_{L^{2}(\Omega)}^{2} .
$$

More $\int_{\Omega} v d x=0$, then according to Poincaré-Wirtinger theorem [20], it comes out that $\|v\|_{H^{1}(\Omega)}^{2} \leq C_{\Omega}\|\partial v / \partial x\|_{L^{2}(\Omega)}^{2}$.

Therefore,

$$
\begin{aligned}
\|v\|_{H^{3}(\Omega)}^{2}= & \|v\|_{H^{1}(\Omega)}^{2}+\left\|\frac{\partial^{2} v}{\partial x^{2}}\right\|_{L^{2}(\Omega)}^{2}+\left\|\frac{\partial^{3} v}{\partial x^{3}}\right\|_{L^{2}(\Omega)}^{2} \\
\leq & C_{\Omega}\left\|\frac{\partial v}{\partial x}\right\|_{L^{2}(\Omega)}^{2}+\frac{1}{2}\left\|\frac{\partial v}{\partial x}\right\|_{L^{2}(\Omega)}^{2} \\
& +\frac{1}{2}\left\|\frac{\partial^{3} v}{\partial x^{3}}\right\|_{L^{2}(\Omega)}^{2}+\left\|\frac{\partial^{3} v}{\partial x^{3}}\right\|_{L^{2}(\Omega)}^{2} .
\end{aligned}
$$

Thus,

$$
\|v\|_{H^{3}(\Omega)}^{2} \leq \max \left(\frac{1}{2}, C_{\Omega}, \frac{3}{2}\right)\left(\left\|\frac{\partial v}{\partial x}\right\|_{L^{2}(\Omega)}^{2}+\left\|\frac{\partial^{3} v}{\partial x^{3}}\right\|_{L^{2}(\Omega)}^{2}\right) .
$$

If we pose

$$
C_{V}=\frac{1}{\left(\max \left((1 / 2)+C_{\Omega}, 3 / 2\right)\right)},
$$

we have

$$
C_{V}\|v\|_{H^{3}(\Omega)}^{2} \leq|\|v\||_{\mathscr{V}}^{2} .
$$

The inegality (A.2) is established and the lemma's proof is completed.

\section{References}

[1] N. E. Huang, Z. Shen, S. R. Long et al., "The empirical mode decomposition and Hilbert spectrum for nonlinear and nonstationary time series analysis," Proceedings of the Royal Society A, vol. 545, no. 1971, pp. 903-995, 1998. 
[2] E. Deléchelle, J. Lemoine, and O. Niang, "Empirical mode decomposition: an analytical approach for sifting process," IEEE Signal Processing Letters, vol. 12, no. 11, pp. 764-767, 2005.

[3] O. Niang, Empirical mode decomposition: contribution à la modé-lisation mathématique et application en traitement du signal et l'image [Ph.D. thesis], University Paris-Est Créteil, Paris, France, 2007.

[4] O. Niang, E. Deléchelle, and J. Lemoine, "A spectral approach for sifting process in empirical mode decomposition," IEEE Transactions on Signal Processing, vol. 58, no. 11, pp. 5612-5623, 2010.

[5] O. Niang, A. Thioune, E. Delechelle, and J. Lemoine, "Spectral intrinsic decomposition method for adaptive signal representation," ISRN Signal Processing, vol. 2012, Article ID 457152, 10 pages, 2012.

[6] O. Niang, A. Thioune, M. C. El Gueirea, E. Deléchelle, and J. Lemoine, "Partial differential equation-based approach for empirical mode decomposition: application on image analysis," IEEE Transactions on Image Processing, vol. 21, no. 9, pp. 39914001, 2012.

[7] F. Catte, P. L. Lions, J. M. Morel, and T. Coll, "Image selective smoothing and edge detection by nonlinear diffusion," SIAM Journal on Numerical Analysis, vol. 29, no. 1, pp. 182-193, 1992.

[8] P. Perona and J. Malik, "Scale-space and edge detection using anisotropic diffusion," IEEE Transactions on Pattern Analysis and Machine Intelligence, vol. 12, no. 7, pp. 629-639, 1990.

[9] Y. Meyer, Oscillating Patterns in Image Processing and Nonlinear Evolution Equations, vol. 22 of University Lecture Series, AMS, Providence, RI, USA, 2002.

[10] S. Osher, A. Sole, and L. Vese, "Image decomposition and restoration using total variation minimization and the H1 norm," Multiscale Modeling and Simulation: A SIAM Interdisciplinary Journal, vol. 1, no. 3, pp. 349-3370, 2003.

[11] M. Lysaker, A. Lundervold, and X. C. Tai, "Noise removal using fourth-order partial differential equation with applications to medical magnetic resonance images in space and time," IEEE Transactions on Image Processing, vol. 12, no. 12, pp. 1579-1589, 2003.

[12] J. Weickert, B. M. ter Haar Romeny, and M. A. Viergever, "Efficient and reliable schemes for nonlinear diffusion filtering," IEEE Transactions on Image Processing, vol. 7, no. 3, pp. 398-410, 1998.

[13] J. Weickert, "A review of nonlinear diffusion filtering," in ScaleSpace Theory for Computer Vision, B. H. Romeny, Ed., vol. 1252 of Lecture Notes in Computer Science, pp. 3-28, Springer, New York, NY, USA, 1997.

[14] D. W. Peaceman and H. H. Rachford, "The numerical solution of parabolic and elliptic differential equations," Journal of the Society For Industrial and Applied Mathematics, vol. 3, no. 1, pp. 28-41, 1955.

[15] D. Barash and R. Kimmel, An Accurate Operator Splitting Scheme for Nonlinear Diffusion Filter, HP Company, 2000.

[16] J. Tumblin and G. Turk, "LCIS: a boundary hierarchy for detailpreserving contrast reduction," in Proceedings of the SIGGRAPH 1999 Annual Conference on Computer Graphics, Los Angeles, Calif, USA, 1999.

[17] G. W. Wei, "Generalized Perona-Malik equation for image restoration," IEEE Signal Processing Letters, vol. 6, no. 7, pp. 165$167,1999$.

[18] Y. L. You and M. Kaveh, "Fourth-order partial differential equations for noise removal," IEEE Transactions on Image Processing, vol. 9, no. 10, pp. 1723-1730, 2000.
[19] J. Tumblin, Private Communication, 2003.

[20] H. Brezis, Analyse fonctionnelle. Théorie et Applications, Masson, Paris, France, 1983.

[21] J. L. Lions, Équations Différentielles Opérationnelles et Problèmes aux Limites, Springer, Berlin, Germany, 1961.

[22] J. D. Murray, Mathematical Biology, Springer, Berlin, Germany, 1993.

[23] P. Flandrin, G. Rilling, and P. Gonçalvés, "Empirical mode decomposition as a filter bank," IEEE Signal Processing Letters, vol. 11, no. 2, pp. 112-114, 2004.

[24] R. Sperb, Maximum Principle and Their Applications, vol. 157 of Mathematics in Science and Engineering Series, Academic Press, New York, NY, USA, 1981.

[25] H. Brezis, Operateurs Maximaux monotones et semigropes de contraction dans les espaces de Hilbert, North-Holland, Amsterdam, The Netherlands, 1972.

[26] G. Aubert and P. Kornprobst, Mathematical Problems in Images Processing, Partial Differential Equations and the Calculus of Variations, vol. 147 of Applied Mathematical Sciences Series, Springer, Berlin, Germany, 2002.

[27] C. Vogel and M. Oman, "Iteration methods for total variation denoising," SIAM Journal on Scientific Computing, vol. 17, no. 1, pp. 227-238, 1996.

[28] G. Engeln-Muellges and F. Uhlig, Numerical Algorithms with C, chapter 4, Springer, Berlin, Germany, 1996.

[29] T. P. Witelski and M. Bowen, "ADI schemes for higher-order nonlinear diffusion equations," Applied Numerical Mathematics, vol. 45, no. 2-3, pp. 331-351, 2003. 

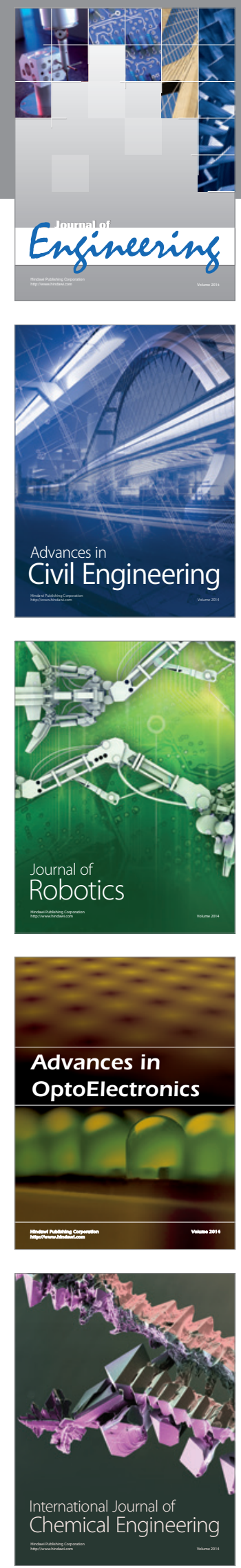

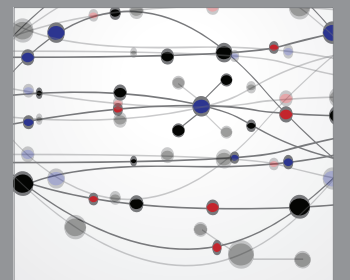

The Scientific World Journal
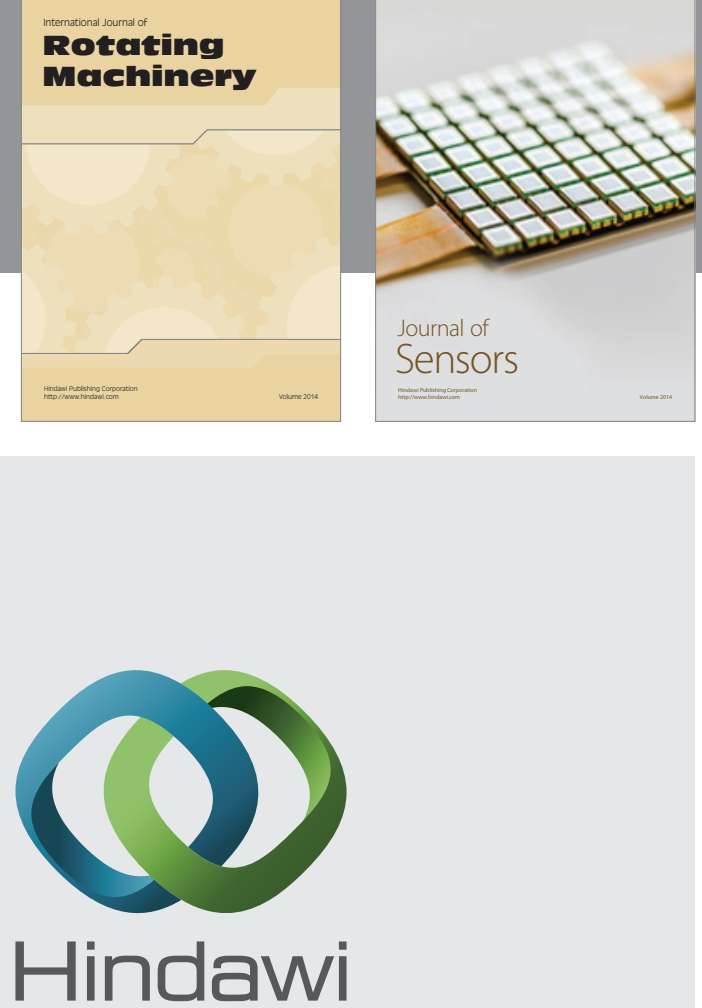

Submit your manuscripts at http://www.hindawi.com
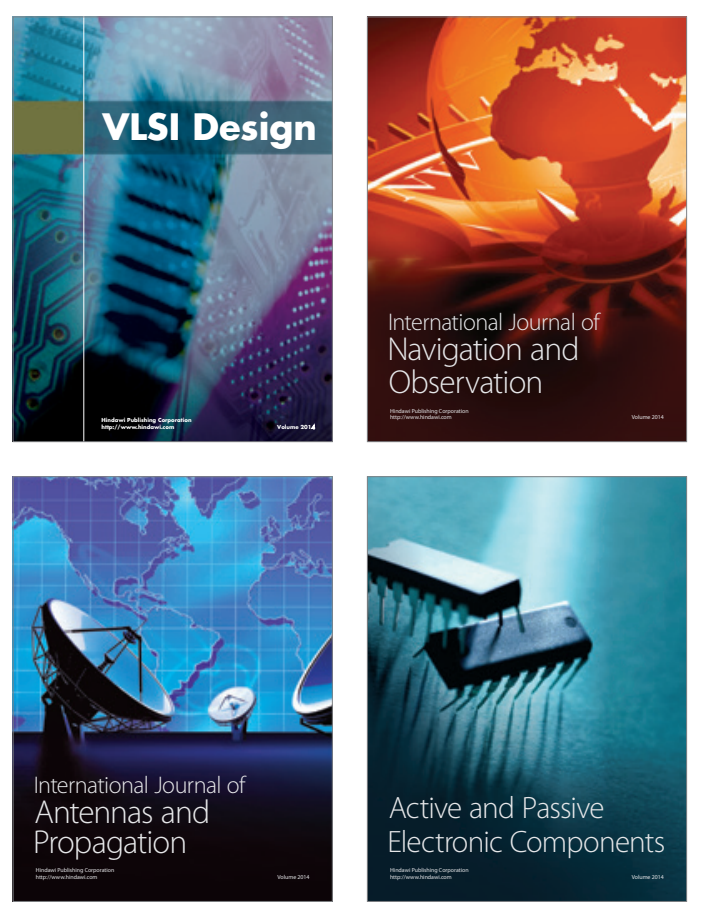
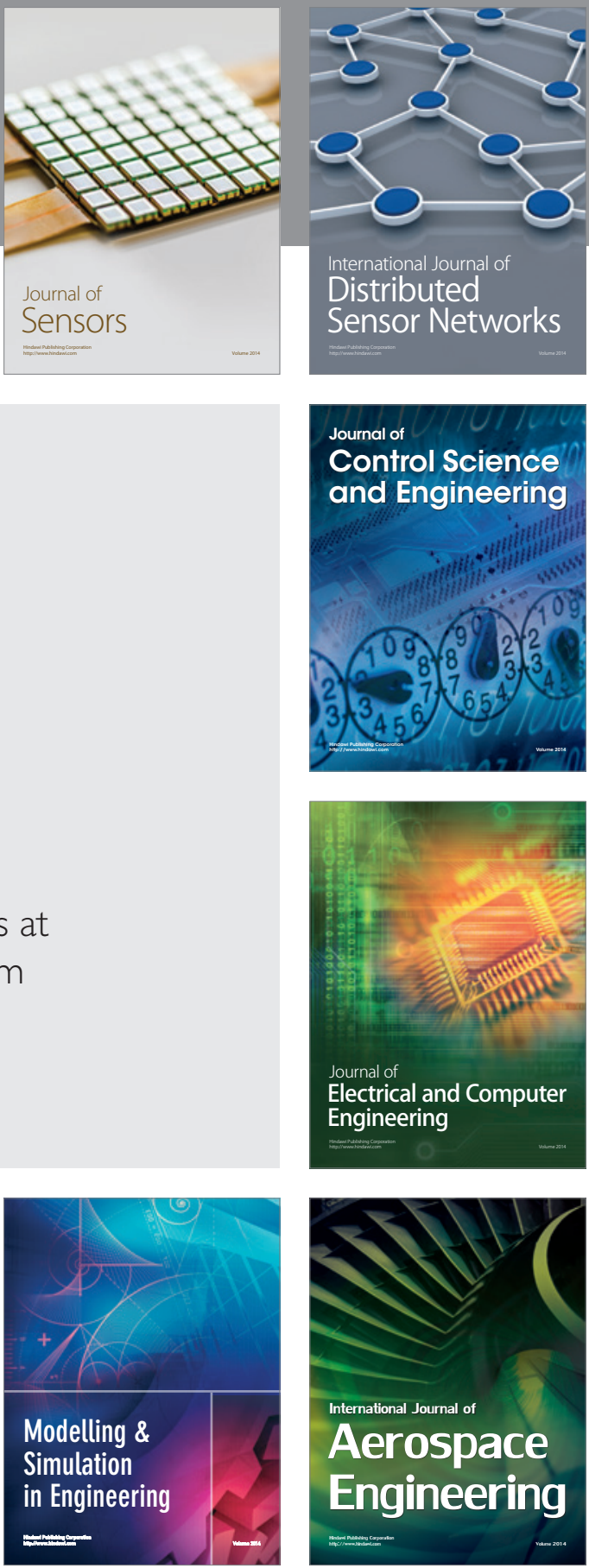

Journal of

Control Science

and Engineering
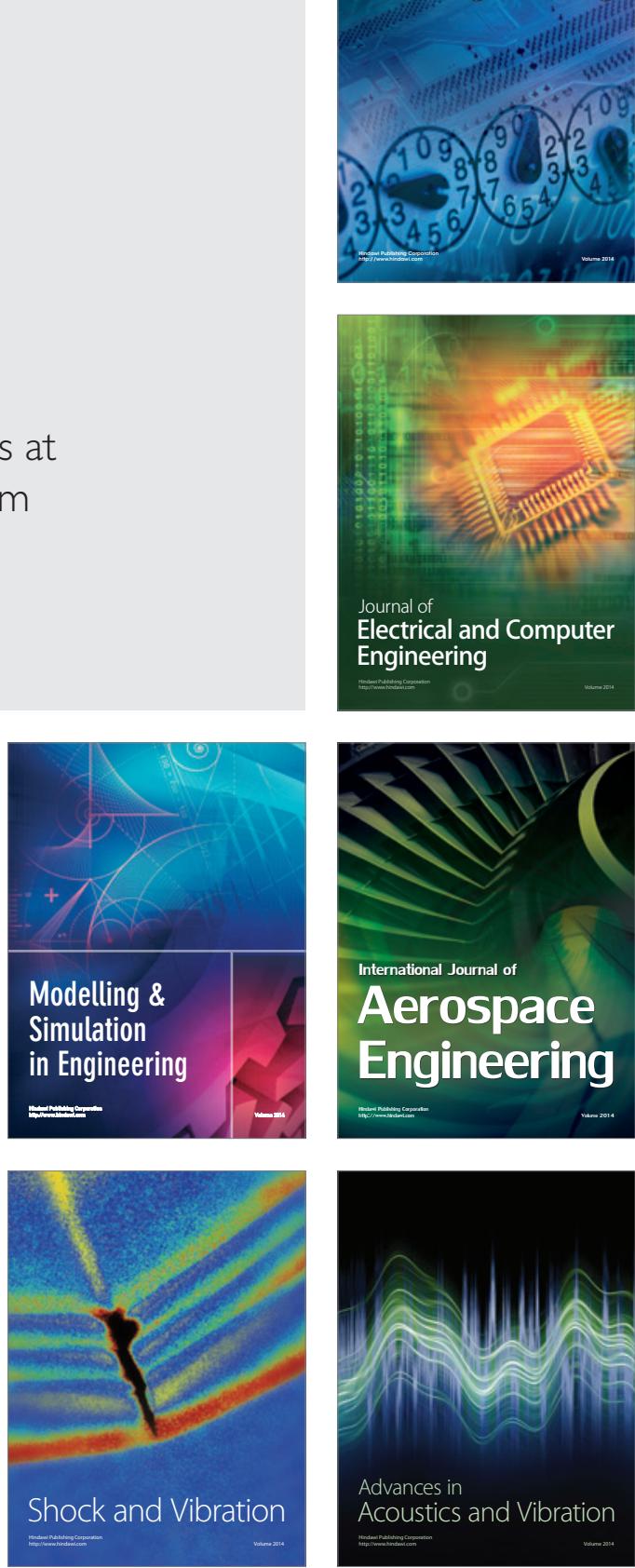